Titre : Crise de confiance et crise de vérité : religion, culture et réticence à la vaccination au Canada

Auteur : Paul Bramadat

Publication : La santé publique à une ère marquée par le doute - Origines religieuses et culturelles de l'hésitation des Canadiens face à la vaccination

Directeurs : Paul Bramadat, Maryse Guay, Julie A. Bettinger et Réal Roy

Pages : $17-50$

ISBN : 978-2-7622-0359-2

URI : http://hdl.handle.net/11143/16026

DOI : https://doi.org/10.17118/11143/16026

Éditeur : Les Éditions de I'Université de Sherbrooke (ÉDUS) 


\title{
Crise de confiance et crise de vérité : religion, culture et réticence à la vaccination au Canada
}

\author{
PAUL BRAMADAT
}

Nous sommes nombreux à supposer ou à espérer que les gens prennent leurs décisions, en matière de santé personnelle, sur la base de "données probantes », en sachant qu'une preuve empirique, correctement interprétée, mènera à une société en meilleure santé, au profit de tous. L'adulte raisonnable et responsable ne serait vulnérable ni à la peur, ni à l'ignorance, ni à la pensée magique, non plus qu'aux allégations d'entreprises égoïstes, de gouvernements arrogants et de groupes d'intérêt nés pour cette seule fin. Les agents libres de toute influence n'existent évidemment que dans l'abstrait. Même une décision aussi simple que de se choisir une nouvelle voiture dépend non seulement de données empiriques facilement accessibles (sur la consommation du véhicule, sur l'espace disponible, etc.), mais aussi d'une foule de variables subjectives (par exemple le pourcentage de son revenu que l'on veut consacrer à une voiture, l'effet de différentes voitures sur le standing social et l'importance que cela peut avoir pour le chauffeur, etc.). L'achat d'une voiture est une entreprise complexe, soumise à des forces psychosociales souvent inconscientes qui incitent une personne à prendre des décisions que d'autres trouveront insensées ou contraires aux faits réels ou au bon goût. En matière de santé personnelle, nombreuses sont les décisions exponentiellement plus complexes, car on reçoit d'amis, de prestataires de soins de santé " alternatifs », de collègues, d' " experts » retrouvés sur Internet et de professionnels de la biologie médicale (qu'on appelle parfois des "médecins allopathes ») des avis discordants, voire équivoques. Cette impression se retrouve non seulement chez les patients ou parents mal informés ou peu intéressés, mais aussi chez les personnes bien informées dont nous parlions précédemment. Les patients reçoivent une avalanche de renseignements conflictuels, mais ils ont souvent bien peu de temps pour prendre des décisions (surtout s'il s'agit de leurs enfants); par ailleurs, ils ont l'impression que les conséquences (pour eux-mêmes, leurs enfants ou la société) seront si graves qu'il devient à la fois extrêmement important et exagérément stressant d'exercer un choix à la fois informé et raisonnable. 
Les variables subjectives qui rendent les décisions au sujet des vaccins si ardues ne proviennent pas seulement de la culture (comme bien des facteurs associés au choix d'un véhicule), mais aussi de la religion. Ceux qui désirent entamer des dialogues efficaces au sujet de l'innocuité des vaccins, de leur coût (modique ou non), de la perception qu'en a le public, du financement, de leurs effets indésirables et de leur efficacité doivent composer, en faisant montre d'un certain esprit critique, mais en toute humilité, avec le rôle puissant que jouent la religion et la culture dans les discours social et médical entourant les pratiques et les politiques d'immunisation. Dans le présent chapitre, je m'interroge sur l'interprétation que pourraient donner les scientifiques, praticiens du secteur de la santé, cliniciens, citoyens et universitaires à la survivance de collectivités réticentes ou opposées à la vaccination et à la pérennité de leur discours, en dépit du consensus scientifique appuyant cette méthode. Je me pencherai d'abord sur les importants mouvements qui redéfinissent le paysage canadien, sous l'angle religieux. Ce portrait sociologique sera, pour le lecteur, une toile de fond grâce à laquelle il saisira comment des cas de refus ou de réticence peuvent être expressément liés à des allégations de nature religieuse ou à des groupes religieux. J'entreprendrai ensuite une brève réflexion critique sur les principales tendances culturelles qui, depuis quelques décennies, ont soulevé des doutes relativement importants sur les programmes de vaccination. Des transformations sociales aussi vastes et probablement irréversibles ont grandement réduit les pouvoirs et les privilèges auparavant attribués à la biologie médicale conventionnelle, en plus de dresser des obstacles au maintien ou à l'expansion de la couverture vaccinale. En troisième lieu, je remettrai en question la distinction généralement convenue entre les motifs religieux et culturels de la réticence et de l'opposition à la vaccination. Bien qu'il y ait de bonnes raisons de faire cette distinction, il serait peut-être bon de déterminer quelle est la perspective commune sur laquelle s'appuient la plupart des arguments religieux et culturels à l'encontre de la vaccination.

\section{La religion au Canada}

Bien des gens disent, au sujet du paysage religieux canadien, que la religion se dirige inexorablement vers son extinction ou que le protestantisme conservateur risque de s'infiltrer dans le discours public; ce sont là néanmoins des impressions biaisées, au sujet de phénomènes plus complexes ou plus répandus. Pour que les lecteurs comprennent mieux les assez rares cas d'opposition officielle (c'est-à-dire d'origine institutionnelle, notamment) à la vaccination pour des motifs religieux et saisissent mieux mon argumentation au sujet de la nature quasi religieuse des plaidoyers des opposants, il serait bon de brosser un portrait des principales tendances associées à la religion dans notre société. Ce chapitre sera donc plutôt descriptif, mais j'exprimerai certaines opinions au sujet des conséquences des tendances relevées dans le secteur canadien des soins de santé, entre autres à l'échelle des programmes de vaccination.

Commençons par cette allégation, devenue un lieu commun chez nous et qui a un certain rôle à jouer dans nos discussions : la religion traverse une mauvaise passe et la spiritualité progresse dynamiquement. Que signifient, dans cette phrase, « religion » et « spiritualité »? Concentrons pour l'instant notre examen sur le débat qui fait rage parmi les spécialistes des sciences religieuses au sujet de la définition de la religion (Arnal, 2000). Pour nous faciliter les choses, donnons à ce terme la définition largement répandue chez les cliniciens, les 
scientifiques, les patients et autres personnes qui s'intéressent à la vaccination. Selon cette définition, la religion englobe doctrines, dogmes, institutions, rites établis, traditions durables, clergé professionnel et gens qui l'appuient, normes en matière de sexualité et de vie éternelle et, généralement, croyance en un dieu. Comme nous l'avons noté dans l'introduction, spiritualité et religion sont souvent juxtaposées et la première renvoie à l'expérience de chacun en matière de théologie ou relativement à la collectivité, à l'univers ou à soi-même. Dans ce schème binaire de réflexion courant, l'expérience spirituelle ne dépend pas entièrement d'un contexte institutionnel religieux. Ce serait plutôt une expérience sublimée et subjective, non rattachée aux institutions et relevant de l'impression?.

Comme le laissent entendre les tableaux qui suivent et des études réalisées depuis vingt ans (Bibby, 2011; Bramadat et Seljak, 2005, 2008, 2013), il est de plus en plus rare que l'on se définisse comme un fidèle des plus importants mouvements protestants chrétiens (c'està-dire anglican, luthérien, presbytérien ou fidèle de l'Église unie). Les catholiques romains semblent résister à cette tendance, mais une visite des grandes églises de Toronto et Montréal confirme que cela est principalement dû à la présence d'immigrants de première génération provenant de pays catholiques comme les Philippines et la Pologne. La tendance déclinante se propagera sans doute également à l'Église catholique, à mesure que les nouveaux arrivants adopteront la démarche des Canadiens de plus vieille souche; en fait, la stabilité relative du nombre des catholiques romains pourrait être modifiée si la politique d'immigration du pays se tournait plus décisivement vers des pays où le catholicisme est moins répandu. Par ailleurs, il est difficile de comprendre ce que veut dire un Canadien français qui se dit catholique (les francophones forment une grande partie de la population catholique canadienne) (Lefebvre, 2008). Dans bien des cas, cette affirmation signifie qu'il a été baptisé selon le rite catholique romain et qu'il continue de considérer le catholicisme comme étant intiment lié à la culture et au nationalisme québécois, bien plus qu'il ne demeure fidèle à ses croyances et pratiques religieuses. Comme de nombreux francophones québécois sont préoccupés par la survie de leur langue, minoritaire dans un continent où habitent près de 350 millions d'anglophones (sans compter ceux dont la langue principale n'est ni l'anglais, ni le français), on remarque chez eux une tendance à percevoir tous les indicateurs d'une culture différente - notamment le français et le catholicisme - comme étant des caractéristiques inaliénables de leur individualité nationale (et peut-être même personnelle) (Lefebvre, 2008). C'est pour toutes ces différentes raisons qu'il vaut mieux être prudent dans l'interprétation de la relative stabilité du nombre de catholiques.

Des processus du même genre, quoique distinctifs, sont en cours dans les populations anglo-protestantes du Canada. En d'autres mots, il existe encore des Canadiens qui, dans un sondage, se disent attachés à l'Église anglicane, à l'Église unie ou à une autre, mais qui dans les faits n'ont aucune relation avec une église, n'ont jamais emmené leurs enfants à l'église et n'entretiennent qu'une relation ténue avec les croyances, modes de pensée, traditions, institutions et écrits de celle-ci. Parmi ces dénominations solidement ancrées dans l'histoire, certaines demeurent dynamiques et connaissent même une certaine croissance, mais il

\footnotetext{
1. Bien que la dyade religion et spiritualité forme un volet important de la plupart des définitions populaires de la religion, la quasi-totalité des formes de la spiritualité soi-disant individuelle est, dans la pratique, étroitement liée aux institutions de tous les types. Par ailleurs, virtuellement toutes les institutions religieuses officielles se disent conçues pour procurer des expériences spirituelles individuelles théoriquement distinctives et pour les promouvoir ou les protéger. Enfin, les concepts sont reliés non seulement sur le plan de la dialectique, au sens étroit, mais aussi dans la vie quotidienne des gens religieux, car elles s'engagent dans des activités qui font appel aux deux forces.
} 
s'agit généralement d'exceptions confirmant la règle; ajoutons même que les réductions constatées depuis trente ans (environ 30 p. cent pour l'Église unie et 20 p. cent pour l'Église anglicane, de 2001 à 2011) pourraient dénoter une crise bien plus grande en réalité. Dans un récent ouvrage traitant de la diversité religieuse et ethnique au Canada, David Seljak et moi avons établi clairement que tous les auteurs s'intéressant aux principales dénominations y avaient observé un discours axé sur la perte (Bramadat et Seljak, 2008), ce qui témoigne que chacune reconnaît son recul progressif en nombre et en prédominance culturelle.

La crise ne se reflète pas uniquement dans cet instrument plutôt lourd que constitue le recensement canadien, dans le cadre duquel on pose à tous les répondants la simple question : "Quelle est votre religion? » Selon l'Enquête sociale générale menée chaque année par Statistique Canada depuis 1985, le nombre de fidèles aux services religieux est en chute prononcée dans tout le Canada, et cela depuis vingt-sept ans. À l'échelle du Canada, à peine 16,9 p. cent des citoyens âgés de quinze ans ou plus ont assisté chaque semaine à un service religieux en 2012, comparativement à 27,2 p. cent en 1985. En 2012 également, 44,1 p. cent des adultes ont déclaré n'avoir assisté à aucun service religieux dans les douze mois précédant le sondage (abstraction faire des mariages, funérailles et baptêmes), comparativement à 29,8 p. cent en 1985 (Statistique Canada, 2014)².

Plusieurs facteurs doivent cependant être considérés avant qu'on ne puisse décrire la situation canadienne actuelle comme un exemple d'une version extrême de la sécularisation - soit que, dans l'ensemble, les groupes religieux perdent leur cohésion institutionnelle, leur attraction pour les personnes et leur emprise sur les leviers de l'État. Premièrement, comme nous le rappelle le sociologue José Casanova (1994), même si la religion ne semble pas intéresser grandement les personnes et encore moins la société en général, elle peut toujours nous surprendre. Dans son ouvrage phare intitulé Public Religions in the Modern World (1994), il a démontré que, dans plusieurs sociétés (Espagne, États-Unis, Brésil et Pologne), les groupes religieux autrefois marginaux se sont rapprochés du pouvoir social et politique et, de cette position avantageuse, ont modifié profondément la société. II est d'ailleurs impossible de comprendre l'élection de Donald Trump, en 2016, sans tenir compte du fait qu'il a obtenu l'appui de plus de 80 p. cent des protestants évangéliques du pays ${ }^{3}$. II serait donc malavisé de supposer que la religion ne sera plus un mode important, sinon prédominant, d'identification et de solidarité politique en Amérique du Nord.

En deuxième lieu, il faut noter que, parmi les dénominations chrétiennes, celles qui sont conservatrices ne subissent pas les mêmes reculs que les autres. Les tableaux ci-dessous montrent qu'elles tirent assez bien leur épingle du jeu, certaines accroissant même leur nombre de fidèles. Un autre sociologue, Reginald Bibby, estime de ce fait que les églises évangéliques réussissent mieux à fidéliser les jeunes et que les valeurs traditionnelles qu'elles mettent de l'avant excellent à les distinguer de la société dominante (1987, 1993, 2011). Par ailleurs, les collectivités protestantes conservatrices ne se définissent ni ne se présentent plus comme avant; nombreuses sont celles qui délaissent l'identification conventionnelle selon

2. Une comparaison aux phénomènes américains figure dans "Religious Service Attendance », Association of Religion Data Archives, consultation le 6 janvier 2017, à l'adresse http://www.thearda. com/quickstats/qs_105. asp. J'aimerais remercier ma collègue Sarah Wilkins-Laflamme, qui m'a fourni les données figurant dans ce paragraphe et m'a aidé à les expliquer.

3. Voir la déclaration officielle de l'Oxford English Dictionary concernant le choix de « post-vérité » comme mot de l'année, le 8 novembre 2016, à l'adresse https://en.oxforddictionaries.com/ word-of-the-year/word-of-theyear-2016. Voir aussi Macdonald (2016) et Shellnutt (2016). 
la dénomination au profit d'appellations plus génériques (comme le " christianisme », le "protestantisme conservateur » ou le «christianisme évangélique »). Quelle que soit la théorie utilisée pour expliquer le relativement bon état de santé des traditions conservatrices dans une société libérale, les statistiques montrent toujours qu'il n'y a pas nécessairement de forces irrésistibles favorisant la sécularisation devant lesquelles la chrétienté sous toutes ses formes doit capituler. La " part de marché », si l'on peut dire, de la chrétienté est passée de 83 p. cent à 67 p. cent de la population de 1991 à 2011 (dernière année pour laquelle Statistique Canada fournit des données officielles). Malgré le fait que cette proportion continue de décliner (on pourrait l'estimer à 60 p. cent environ en 2017), la chrétienté demeure, et de loin, le plus important groupe religieux au Canada. À l'opposé, en 2017, toutes les religions non chrétiennes (islam, judaïsme, hindouisme, sikhisme, bouddhisme), réunies, ne rassemblaient qu'environ 10 p. cent de la population. Si l'on demandait à chacun d'affirmer (ou de cesser d'affirmer) qu'il est un fidèle de la religion pentecôtiste ou baptiste ou même qu'il est catholique, anglican ou presbytérien, cela remettrait en question les affiliations traditionnelles basées sur la dénomination et cela permettrait de tirer des conclusions moins négatives sur l'avenir de la chrétienté au Canada, même si les renseignements disponibles laissent croire que certaines dénominations (anglicans et chrétiens de l'Église unie) sont en réel danger d'extinction (voir le tableau 1.1).

Tableau 1.1 Déclin ou croissance des principales dénominations chrétiennes depuis 1991

\begin{tabular}{|l|l|l|l|}
\hline Dénomination & $\mathbf{1 9 9 1 - 2 0 0 1}$ & $\mathbf{2 0 0 1 - 2 0 1 1}$ & Total en 2011 \\
\hline Catholicisme & $+5 \%$ & $-1 \%$ & 12500000 \\
\hline Église unie & $-8 \%$ & $-29,5 \%$ & 2000000 \\
\hline Anglicanisme & $-7 \%$ & $-20 \%$ & 1500000 \\
\hline Église luthérienne & $-4,5 \%$ & $-21 \%$ & 478000 \\
\hline Protestantisme conservateur & $+10 \%$ & $+19,5 \%$ & 4200000 \\
\hline Église baptiste & $+10 \%$ & $-13 \%$ & 635000 \\
\hline Église pentecôtiste & $-15 \%$ & $+30 \%$ & 478500 \\
\hline Églises chrétiennes n.s.a.* & $+154 \%$ & $+170,5 \%$ & 1500000 \\
\hline
\end{tabular}

* «Églises chrétiennes non spécifiées ailleurs ». On inclurait dans ce groupe les personnes pratiquant une forme de christianisme associée au protestantisme le plus conservateur et qui ne sont pas affiliées à une dénomination actuelle reconnue, ainsi que les chrétiens dont la fidélité aux traditions n'est guère plus que symbolique.

Tableau 1.2 Croissance du nombre de personnes ne s'identifiant à aucune religion depuis 1991

\begin{tabular}{|l|l|l|}
\hline Identité non religieuse & 1991 & $\mathbf{2 0 1 1}$ \\
\hline Aucune religion & $3390000(12 \%)^{*}$ & $7850000(24 \%)$ \\
\hline Agnostiques & 22000 & 36000 \\
\hline Athées & 13500 & 49000 \\
\hline
\end{tabular}

* Pourcentage de la population canadienne

Troisièmement, on ne sait pas encore comment interpréter l'accroissement considérable de personnes « ne pratiquant aucune religion » dans le paysage démographique religieux canadien. Les personnes qui se décrivent ainsi dans les sondages ne forment pas en effet qu'un segment assez populeux de la société canadienne (environ 24 p. cent de la population entière), car leur nombre s'accroît à un rythme remarquable (il est passé de 12 à 24 p. cent de la population entre 1991 et 2011 et devrait avoisiner les 30 p. cent en 2017, selon le tableau 1.2). Signalons toutefois que cette catégorie englobe des gens de toutes sortes: 
athées, agnostiques, humanistes séculiers, chrétiens conservateurs rejetant les appellations conventionnelles basées sur la dénomination (mais tout de même intensément engagés dans leur foi), personnes estimant que l'État ne devrait pas s'intéresser à leur identité religieuse et différents adeptes de la spiritualité du nouvel âge pour qui tout ce qui est associé à la religion est négatif, mais qui ne trouvent ni dans le formulaire du recensement, ni dans le discours public une option qui leur convient ${ }^{4}$. De toute évidence, le nombre croissant de gens « sans religion » dans notre société n'augure pas d'une fin prochaine, en ce qui concerne les questions, habitudes et pratiques souvent associées à la religion, mais la signification de leur présence n'est pas encore clairement établie.

Enfin, les groupes religieux musulmans, bouddhistes, sikhs, hindous et chinoistraditionnels ${ }^{5}$ augmentent également en nombre au Canada (voir le tableau 1.3). Les quatre premiers se sont accrus de 50 à 130 p. cent dans chacune des dernières décennies (Bramadat et Seljak, 2008; Statistique Canada, 2011) et on assiste actuellement à une renaissance de la spiritualité autochtone. À moins que le Canada ne modifie radicalement ses politiques d'immigration et que le taux de natalité des Autochtones ne diminue dans une même proportion, le nombre des personnes et des collectivités associées à ces religions et ces formes de spiritualité continuera sans doute à augmenter, en chiffres absolus et relatifs.

Tableau 1.3 Portrait empirique des religions au Canada, entre 1991 et 2011*

\begin{tabular}{|l|l|l|}
\hline Religions & 1991 & $\mathbf{2 0 1 1}$ \\
\hline Musulmans & 253000 & 1050000 \\
\hline Sikhs & 147000 & 454000 \\
\hline Hindous & 157000 & 500000 \\
\hline Juifs & 318000 & 329000 \\
\hline Bouddhistes & 163000 & 367000 \\
\hline Aucune religion & 3390000 & 7850000 \\
\hline
\end{tabular}

* Vu l'importance du nombre de chrétiens (au-delà de 20 millions de personnes), il est utile de connaître l'évolution considérable du pourcentage de Canadiens disant appartenir à cette religion. En 1991, 83 p. cent des Canadiens se déclaraient chrétiens, contre 67 p. cent en 2011.

Les quelques observations finales suivantes devraient indiquer quel est le lien entre le paysage religieux changeant et les enjeux dont traite le présent ouvrage. Quatre grandes tendances, se dégageant quasi simultanément, signifient que nous assistons à la fin de plusieurs siècles d'une prédominance non contestée du christianisme au Canada : 1) la fin

4. En effet, lorsque je réalise des sondages informels et anonymes auprès de mes étudiants inscrits au baccalauréat, il s'en dégage des tendances intéressantes témoignant de l'évolution au sein de la société en général : à peine 20 p. cent d'entre eux ont été éduqués dans le respect d'une perspective religieuse formelle couvrant le monde dans son entier et entre 50 et 60 p. cent se disent « non religieux». Parmi ceux-ci, cependant, 85 p. cent environ se livrent à des pratiques qu'ils disent « spirituelles » (yoga, méditation, prière, etc.) et entre 60 et 70 p. cent seraient d'accord avec cette phrase : "Je pratique la spiritualité, mais ne fais partie d'aucune religion. "

5. La cohorte des personnes d'origine chinoise qui habitent au Canada et qui sont religieuses est assez importante, mais les techniques employées actuellement par Statistique Canada ne réussissent pas à la délimiter suffisamment. C'est pourquoi celles dont la vie religieuse comporte la piété filiale, la vénération des ancêtres, des visites occasionnelles au shaman, aux praticiens de la médecine chinoise et à des églises chrétiennes et des offrandes à des représentations domestiques ou publiques de Bouddha se retrouvent souvent classées parmi les bouddhistes, les chrétiens ou les personnes sans religion. Ces catégories n'arrivent pas à décrire suffisamment leur vie religieuse. Comme il y a plus d'un million de Canadiens d'origine chinoise (dont beaucoup de récents immigrants), il est déplorable que nous n'ayons pas encore trouvé un moyen de décrire leur vie religieuse, souvent assez complexe, dans toutes ses dimensions. Voir Paper, Paper et Lai (2009). 
graduelle d'une pratique voulant que l'on soit fidèle à une seule affiliation religieuse tout au long de sa vie; 2) les changements démographiques toujours en cours en raison des politiques d'immigration canadiennes; 3) l'apparition de nouvelles formes de sécularisation au Canada et 4) l'accroissement du nombre de personnes qui refusent de s'affilier à une religion. II reste à savoir quel sera l'avenir des structures, croyances, documents, pratiques et collectivités de la religion chrétienne dans la société canadienne; il est trop tôt pour conclure que le déclin actuel se transformera en extinction. Comme les immigrants chrétiens constituent encore une part importante des nouveaux venus accueillis chaque année au Canada (Statistique Canada, 2003) et que la cohorte des arrivants européens d'origine chrétienne demeure bien assise, avec ses coutumes, dans pratiquement toutes nos institutions, la chrétienté conservera son rôle important dans la société canadienne dans l'avenir prévisible. Il y a moins de certitude en ce qui concerne la manière qu'adopteront à l'avenir les chrétiens canadiens pour pratiquer leur religion, s'y identifier, croire à ses principes, se rassembler et dialoguer avec les nonchrétiens et l'État.

L'analyse qui précède devrait en conséquence nous amener à douter de l'allégation si répandue selon laquelle la religion est en déclin et la spiritualité est en hausse. La réponse dépend en grande partie de ce que l'on entend par religion et spiritualité. Si l'on donne de la première une définition élargie, englobant non seulement les dénominations et institutions officielles (dont un bon nombre sont en déclin), mais aussi la multitude de mouvements « spirituels » réels ou en démarrage qui existent et se développent à l'intérieur ou à l'extérieur de nos institutions (Woodhead et Heelas, 2005; Beaman et Beyer, 2008; Wuthnow, 2000), on pourra dire que la religion se débrouille assez bien dans notre société.

\section{Religion, vaccination et culture canadienne}

Ayant étudié certains des aspects contemporains les plus pertinents de la religion au Canada, j'ai observé, comme bien d'autres auteurs du présent ouvrage, que l'hésitation face à la vaccination semble être plus fortement reliée à une culture à la structure relativement amorphe qu'à des motifs religieux précis (une distinction que je remets en question plus loin) (v. Kata, $2010,1713)$. En fait, bien que les interdits religieux officiels aient été rares et bien espacés dans le temps, on en compte une bonne quantité et ils peuvent avoir des effets non négligeables. Toute éclosion d'une maladie devrait nous amener à réfléchir aux politiques de santé publique conventionnelles (Salmon et coll., 1999, 47) et aux convictions et sensibilités propres aux collectivités touchées au départ par l'infection. Le College of Physicians of Philadelphia (2017) a réussi à cerner les deux principales préoccupations religieuses associées à la vaccination : «1) les dilemmes éthiques associés à l'utilisation de cellules humaines pour créer des vaccins et 2) la perception que le corps humain est sacré, ne devrait pas recevoir de produits chimiques ou de sang ou tissus d'origine animale et que la guérison devrait provenir de Dieu ou de la nature » (v. Grabenstein, 2013) ${ }^{6}$. Parmi les exemples les plus frappants (dont il sera question au chapitre 8 du présent ouvrage, sous la plume de Bettinger et MacDonald) de flambées de maladies liées à la religion, notons les éclosions de rougeole en 2014 et de coqueluche en 2012, toutes deux ayant eu lieu dans la vallée du Fraser, en Colombie-Britannique, ainsi que celle de la rubéole de 2005, à proximité de Woodstock, en Ontario (v. Alphonso, 2012; Basrur,

6. Voir College of Physicians of Philadelphia (2017). 
2005; Maki, 2014; De Serres et coll., 2013; Deeks et coll., 2014). Bien qu'il existe dans ces cas des différences à l'échelle des maladies, des régions et des collectivités religieuses intéressées, un petit nombre de protestants fondamentalistes a déclaré que les vaccins s'interposent dans les plans établis par Dieu pour chaque personne et équivalent donc à une tentative de l'Homme d'outrepasser l'autorité divine". Les personnes et les groupes intéressés à ces récents cas au Canada sont affiliés au mouvement réformiste néerlandais, né de la Réforme protestante. Celle-ci, organisée sous la direction de l'évangéliste suisse Jean Calvin, a gagné plusieurs sociétés européennes au cours des siècles suivants et s'est adaptée aux milieux politiques et nationaux dans lesquels elle évoluait. Un mouvement associé à ce protestantisme originaire des Pays-Bas existe au Canada depuis le XIXe siècle et son importance s'est accrue avec la seconde vague d'immigration néerlandaise ayant suivi la Deuxième Guerre mondiale. Une partie de ce groupe doute toujours des agissements du monde laïque dominant, à l'instar de ses ancêtres européens. Bien que les fidèles n'habitent pas en communauté ni ne s'isolent du monde (comme le fait la communauté amish), certains ont adopté une perception du monde et de la suprématie divine qui les amène au non-conformisme vis-à-vis de l'État et de la société. Cet antagonisme par rapport aux forces sociales et religieuses hégémoniques qui les entourent s'est amorcé au XVI ${ }^{\mathrm{e}}$ siècle, dans une période marquée par des conflits avec autant l'Église catholique que l'Église luthérienne (et par des querelles intestines). L'insistance initiale des Réformistes sur la prédestination les a isolés des autres religions et est devenue une de leurs caractéristiques fondamentales. Une telle approche du monde a incité des membres de ces groupes à s'insurger contre toute intervention humaine susceptible d'entrer en conflit avec d'importants éléments du "plan divin » régissant leur existence. Pour les personnes ayant adopté une version aussi stricte de cette croyance - dont le nombre ne fut jamais élevé, même après l'expansion du protestantisme réformateur -, l'Homme ne peut agir au détriment du plan évident que Dieu a conçu à son endroit. Parmi elles, certaines feront leur une position aussi conservatrice sur le leadership des femmes, sur la consommation de boissons alcoolisées, sur l'homosexualité et sur l'activité sexuelle avant le mariage. De telles opinions sont évidemment conformes à bien des vues du protestantisme fondamentaliste (Bramadat, 2000; Stackhouse, 1993); ce qui, pour nos collègues œuvrant en santé publique, rend intéressante la sous-culture réformiste d'origine néerlandaise, c'est que, pour certains de ses fidèles, les vaccins sont un obstacle moralement répréhensible au plan divin.

Ce groupe n'est évidemment pas le seul, parmi les mouvements chrétiens, à s'inquiéter de la vaccination. Certains catholiques, par exemple, n'acceptent pas que différents vaccins aient été fabriqués au moyen de lignées cellulaires extraites de fœtus humains après un avortement ${ }^{8}$. Les représentants officiels de la hiérarchie catholique ne veulent pas appuyer des interventions médicales faisant appel à des lignées cellulaires obtenues grâce à l'avortement même celles qui ont eu lieu il y a des dizaines d'années et n'étaient pas reliées aux programmes d'immunisation -, mais ils reconnaissent, semble-t-il, qu'en l'absence de solutions autres que la vaccination, on doit prévenir les souffrances inutiles et cela rend la vaccination des enfants non seulement acceptable, mais nécessaire. Dans l'article intitulé « Moral Reflections on

7. Pour des déclarations du même genre, se reporter à Kennedy et Gust (2008), Offit (2011), Oostvogel et coll. (1994), Ruijs et coll. (2011) et Spier (2001). Voir également « Vaccines: A Religious Contention », publié par le Vaccine Risk Awareness Network (consulté le 7 janvier 2017, à l'adresse http://vaccineriskawareness.com/ Vaccines-A-Religious-Contention-.

8. Voir «Infant Immunization The Catholic Parents' Guide », par Donald J. Henz, consulté le 7 janvier 2017, à l'adresse https://cogforlife.org/catholicguide.pdf. Henz est associé au groupe Enfants de Dieu, qui se proclame leader mondial des opposants à l'avortement et dirige la campagne de promotion des vaccins, des pratiques médicales et des produits de consommation respectueux de l'éthique. 
Vaccines Prepared from Cells Derived from Aborted Human Foetuses » (2005), l'Académie pontificale pour la vie en arrive à la conclusion suivante:

En ce qui concerne les maladies pour lesquelles il n'existe pas d'autres vaccins acceptables sur le plan de l'éthique, on est en droit de les refuser si cela est possible sans causer de risque important pour la santé des enfants et, indirectement, pour la population en général. Par contre, en présence d'un tel risque, les vaccins posant des problèmes moraux peuvent être utilisés sur une base temporaire. Moralement, on peut procéder ainsi car le devoir d'éviter la coopération matérielle passive s'élimine en présence d'aussi grands inconvénients. De tels cas font également ressortir un motif proportionnel, à savoir qu'il faut accepter l'utilisation de ces vaccins pour pallier le danger d'expansion de l'agent pathogène attribuable à la non-vaccination des enfants. La vaccination contre la rubéole en est un exemple frappant. (546)

En d'autres mots, les autorités catholiques reconnaissent ainsi que l'immunisation contre la rubéole, produite par l'utilisation de lignées cellulaires de deux fœtus avortés (l'un datant de 1964 et l'autre de 1970), permet d'éviter notamment l'avortement spontané ou provoqué de nombreux fœtus, ainsi que la souffrance infligée aux nourrissons et mères qui deviendraient vulnérables à l'infection. Pour ces différentes raisons et malgré le fait que des catholiques ont d'importantes réserves au sujet de la méthode d'élaboration des vaccins, il nous a été impossible de repérer, au Canada, des flambées de maladies pouvant être attribuées à des déclarations de catholiques attachés aux traditions ou plus conservateurs encore au sujet des vaccins et de la santé (v. Grabenstein, 2013, 1999; CDC, 1999).

J'ajouterais, outre l'expression conventionnelle d'objections religieuses formelles, une troisième possibilité, d'un ordre plus explicitement politique : dans certains cas, les leaders culturels ou religieux considèrent les vaccins comme une forme d'impérialisme. Certains leaders musulmans nigérians, par exemple, ont refusé que leur population soit vaccinée contre la poliomyélite, en invoquant la possibilité que le vaccin ait été délibérément contaminé par des composants favorisant l'infection par le VIH et l'infertilité (Jegede, 2007; UNICEF, 1997)', une rumeur que des leaders religieux ont aussi répandue en partie au Cameroun (FeldmanSavelsberg et coll., 2000).

Par ailleurs, il n'est pas surprenant que certains leaders religieux et politiques de sociétés musulmanes remettent en question la valeur des programmes de vaccination; après tout, notamment en Afghanistan et au Pakistan, les milieux internationaux de santé publique n'ont-ils pas été alarmés lorsqu'il a été dévoilé que des employés de la Central Intelligence Agency des États-Unis, désireux de localiser Oussama ben Laden, s'étaient fait passer pour des travailleurs occidentaux livrant des vaccins? Certains, sur place, se seront sans doute réjouis de la mort de ben Laden en mai 2011, mais la situation a semé le doute chez d'autres, quant aux motifs pouvant sous-tendre la participation occidentale à des projets de soins de santé en terre étrangère. À tel point qu'en 2012, des bénévoles chargés de la vaccination

9. On peut lire, dans Jegede :

Dans un article publié sur le site de nouvelles sud-africain News24.com, Sule Ya'u Sule s'exprime ainsi, pour le compte du gouverneur de Kano: "Depuis le 71 septembre, le monde musulman commence à se méfier de tout geste du monde occidental... Nos gens sont très préoccupés par le vaccin contre la poliomyélite. » Dans le même article, Datti Ahmed, médecin de Kano dirigeant un puissant groupe musulman appelé Supreme Council for Sharia in Nigeria (SCSN), est cité également; selon lui, les vaccins contre la polio " sont impurs et altérés par de méchants Américains et leurs alliés occidentaux». Ahmed poursuit en ces termes: "Nous croyons que des Hitler modernes ont délibérément altéré les vaccins oraux contre la polio avec des médicaments qui rendent infertiles et... des virus dont on sait qu'ils causent le VIH et le sida. » $(2007,478)$ 
contre la poliomyélite en Afghanistan furent assassinés par les talibans, ce qui a entraîné, au bout de quelques mois, l'abandon de la campagne dans certaines provinces pour des raisons de sécurité; la population estimait en effet que l'intervention était agressive, impérialiste et contraire aux pratiques musulmanes (Graham-Harrison, 2012, 2013; v. également Warraich, 2009). Autrement dit, il faut considérer les controverses publiques et politiques suscitées par la vaccination et, au sens plus large, par le rôle des milieux militaires et médicaux occidentaux dans les pays en développement dans le contexte d'une longue histoire d'impérialisme de "l'Occident » sur le « monde musulman » et même sur le " monde en développement ». Lorsqu'on parle de ces intervenants et de ces domaines d'activité, on se fonde sur des notions relatives à l'Occident et au monde musulman qui font de réalités complexes et surdimensionnées des « communautés imaginées » convenant mieux à la narration et à la politisation et l'on peut déplorer la banalisation d'une réalité plus complexe; il n'en demeure pas moins que les soupçons entourant les interventions médicales occidentales, telles que la vaccination, étaient prévisibles. C'est probablement pourquoi le titre original d'un éditorial de la revue Scientific American de mai 2013, qui traitait des conséquences de l'assassinat d'Oussama ben Laden, était : « Les espions qui ont saboté la santé mondiale ${ }^{10}$ ».

Dans la plupart des cas, quand une communauté religieuse officielle - ou seulement ses leaders - refuse la vaccination ou éprouve de graves doutes à cet égard, les intervenants sont le plus fréquemment des «fondamentalistes » qui présentent une version très réduite et très conservatrice de traditions plus étendues (généralement en faveur de la vaccination) et qui disent appartenir à des communautés assiégées, souvent par une société dominante. Notons toutefois qu'outre le petit nombre de catholiques préoccupés par certains aspects de la vaccination, d'autres groupes non fondamentalistes, comme les fidèles du mouvement de la Science chrétienne (dont le nombre ne dépasse probablement pas 5000 au Canada) sont depuis longtemps opposés à la vaccination. Dans ce cas en particulier, l'opposition ou la réticence n'émanent pas d'une relation conflictuelle généralisée avec une société libérale hostile ou corrompue. Elles proviennent plutôt d'une conviction religieuse, à savoir que la prière est supérieure à la médecine quand il s'agit de guérir le corps, car ce dernier et la maladie sont des réalités illusoires, à l'instar de la matière. La prière est le moyen par lequel les croyants s'affranchissent des contraintes (comme la maladie) que leur impose le monde matériel. Cette façon de voir la vaccination découle donc d'une perspective religieuse qui remet en question la maladie, la santé, voire la réalité dans son ensemble, mais n'empêche pas les fidèles d'évoluer assez facilement dans la société libérale (Stark, 1998).

Les sociologues ont noté depuis longtemps que les gens choisissent les éléments de la doctrine religieuse qu'ils appliquent selon leurs propres préférences et souvent de manière apparemment contradictoire (Bibby, 1987, 1993). En 2017, cette observation ne surprend plus les spécialistes des sciences religieuses, mais étonne encore les profanes et irrite les partisans de l'orthodoxie. Quoi qu'il en soit, on pourrait extrapoler légèrement, à partir de cette tendance, et suggérer que les initiés manifestent autant de flexibilité dans leur manière de composer

10. Voir « How the CIA's Fake Vaccination Campaign Endangers Us All », dans Scientific American, 2013, consulté le 7 janvier 2017, à l'adresse https://www.scientificamerican.com/article/how-cia-fake-vaccination-campaignendangers-us-all/; se reporter également à Saeed Shah, « CIA Organized Fake Vaccination Drive to get Osama bin Laden's family DNA », The Guardian, 11 juillet 2011, à l'adresse 
avec les effets des traditions religieuses sur les pratiques médicales telles que l'immunisation". Dans certaines situations, la position officielle des groupes religieux à l'égard de la vaccination n'a pas évolué au même rythme que la recherche et l'évolution des politiques publiques, mais dans d'autres circonstances, on se montre plus pragmatique quand on en discute. Le Vatican, par exemple, autorise maintenant la vaccination contre la rubéole et déclare que les enfants nés et à naître ne doivent pas payer le prix de la lutte légitime visant les fabricants de médicaments qui produisent des vaccins moralement répréhensibles en utilisant des lignées cellulaires (mais non les cellules ou les tissus) de fœtus avortés (Glatz, 2005).

À ces cas précis d'opposition ou de réticence attribuable à des motifs religieux, nous pourrions ajouter au moins deux autres situations plus générales se rapportant à l'analyse qui précède et auxcontroverses entourant la vaccination. Premièrement, la politique d'immigration canadienne étant fondée sur un système à base de points qui avantage les candidats connaissant les normes, les langues et le système d'éducation occidentaux, les nouveaux arrivants sont déjà, en grande partie, acquis au modèle de la médecine occidentale. Bien que l'on commence à peine à réaliser des études sur l'immigration et les attitudes à l'égard des vaccins (v. Streefland, 2001)12, les Canadiens qui, en nombre croissant, sont assez récemment arrivés d'Asie, d'Afrique et d'Amérique latine apportent avec eux des perceptions du corps, de la maladie, de la collectivité et de la médecine (Coward et Ratanakul, 1999) différentes de celles de la société dominante. Il est inutile (et probablement contraire à la déontologie) d'insister pour que ceux qui croient à une médecine alternative ou complémentaire d'origine autre qu'occidentale abandonnent ces principes à leur arrivée ici; on ne peut s'y attendre, ni même l'espérer. Cependant, les partisans de la vaccination qui veulent à la fois établir une relation respectueuse avec une cohorte qui grandit au sein de nos sociétés et promouvoir l'immunisation auprès de ces sous-cultures doivent absolument s'informer de l'évolution des démarches alternatives ou autres qu'occidentales, notamment la médecine ayurvédique, la médecine chinoise traditionnelle et l'acupuncture.

En second lieu, l'accroissement du nombre de citoyens ne pratiquant aucune religion pourrait présenter quelques défis aux partisans de la vaccination. Bien que les tendances dégagées des sondages (voir précédemment) portent à croire qu'en 2017, au-delà de 30 p. cent des Canadiens ne déclareront aucun affiliation religieuse, bien des gens faisant partie de cette cohorte déstructurée pourraient fort bien appartenir à la sous-culture des personnes non religieuses professant une certaine spiritualité (Chandler, 2008). Les membres de cette sousculture expérimentent la spiritualité et accordent de la valeur à leur expérience (quoique celleci soit vaguement définie), mais ils ne ressentent aucun besoin de l'associer ou de s'associer

11. Voir Grabenstein (2013). L'approche ecclésiastique en matière d'immunisation est plutôt complexe; voir Vitello (2010) et "Cultural Perspectives on Vaccination », dans The History of Vaccines, dernière modification le 15 décembre 2014, à l'adresse http://www. historyofvaccines.org/content/articles/cultural-perspectivesvaccination; « What Is Christian Science? » Christian Science, consulté le 8 janvier 2017, à l'adresse http://www. christianscience.com/what-is-christian-science; "Outbreak of Measles Among Christian Science Students Missouri and Illinois, 1994," Centers for Disease Control and Prevention, jer juillet 1994, à l'adresse https://www. cdc.gov/mmwr/preview/mmwrhtml/00031788.htm.

12. En ce qui concerne le rapport entre les politiques d'immigration canadiennes et nos niveaux d'immunisation, il serait sage de se rappeler l'observation de Streefland (2001) : "On migre et on communique de plus en plus et cela signifie qu'en matière de santé, les définitions et les explications utilisées partout dans le monde ne sont plus isolées les unes des autres, mais plutôt le résultat de processus de diffusion et d'échange mondiaux et d'interprétation locale. »Cela apparaît clairement dans le cas de l'immunisation; il suffit de penser aux messages d'éducation sanitaire qui sont préparés à Genève ou à Atlanta, qui se rendent jusqu'aux mères malgré l'éloignement grâce à des interventions de travailleurs de la santé formés pour le travail en ville et sont ensuite interprétés de manière à ce qu'ils s'intègrent dans des théories locales sur la contagion et les maladies contagieuses. » (162). 
eux-mêmes à une religion officielle. En passant, au bout de plus de quinze ans d'enseignement dans deux universités canadiennes, j'ai calculé qu'environ la moitié de mes étudiants se sont décrits comme appartenant à cette sous-culture; les recherches faites aux États-Unis et au Canada, ainsi que les reportages récents dans les journaux canadiens, confirment cette tendance ${ }^{13}$. Quoique les sociologues commencent à peine à soumettre la cohorte canadienne à l'analyse (Chandler, 2010), cette approche s'étend rapidement et pourrait fort bien l'emporter sur la foi chrétienne dominante d'ici dix à vingt ans, notamment dans les grands centres urbains comme Vancouver, Toronto et Montréal. Conformément à sa définition, elle regroupe des gens dont on s'attend à ce qu'ils se dissocient progressivement, voire en arrivent à se méfier des institutions religieuses. II n'est pas déraisonnable de s'imaginer que cette méfiance pourrait éventuellement s'appliquer à différents genres d'institutions officielles. Aucune étude empirique des perceptions de ces personnes au sujet de la vaccination n'a encore été réalisée, mais des données récentes laissent entrevoir que cette sous-culture en progression rapide pourrait être composée, en nombre disproportionné, d'opposants et de réticents. D'ailleurs, comme le note Kata (2010), on observe un certain chevauchement entre les notions de spiritualité et de modes de vie alternatifs et les arguments des opposants à la vaccination : «La plupart (soit 88 p. cent des sites Web analysés), écrit-elle, jugent que le traitement par les plantes, I'homéopathie, la chiropratique, la naturopathie et l'acupuncture sont supérieurs à la vaccination. Le motif en est qu'il faut revenir à la nature (selon 88 p. cent des sites) et que les méthodes naturelles de guérison, y compris l'allaitement, l'absorption d'aliments complets et une expérience naturelle de la maladie chez l'enfant, sont préférables » (1712; v. Chandler, 2008, 2010; Guay et coll., 2009). Comme plusieurs de nos coauteurs le proposent, il serait sage, pour les partisans de la vaccination, de porter une attention particulière aux nouvelles recherches en sciences sociales qui traitent des personnes non religieuses professant une certaine spiritualité, afin de mieux comprendre comment elles envisagent des pratiques comme l'immunisation et comment, en conséquence, les travailleurs de la santé pourraient interagir avec elles d'une manière informée, respectueuse et pragmatique.

\section{Motifs d'opposition culturels}

Ayant décrit le contexte évolutif permettant de comprendre les allégations de nature expressément religieuse portées à l'encontre de la vaccination au Canada, je me pencherai maintenant sur les grandes tendances qui sous-tendent l'expansion de l'opposition et de l'hésitation face à la vaccination qui sont plus couramment motivées par la culture.

Examinons d'abord ce qui pourrait être appelé la crise de confiance.

Bien que la plupart des gens, dans notre société, accordent à la vaccination les mérites qui lui reviennent, on ne peut certainement pas dire que chaque personne de l'imposante cohorte qui partage cet avis - soit plus de 80 p. cent de la population, pour la plupart des vaccins (UNICEF/OMS, 2012) - a analysé avec soin et dans un esprit critique les données empiriques qui appuient la vaccination et celles qui la condamnent. Ce serait plus juste de dire que nous avons confié cette analyse critique à des tiers. En d'autres mots, la plupart d'entre

13. Voir Bibby (2011) et Davison (2013), de même que : "Nones' on the Rise », Pew Research Center, 9 octobre 2012, à l'adresse http://www.pewforum.org/2012/10/09/nones-on-the-rise/. Dans cette étude, on conclut que 37 p. cent des Américains pourraient faire partie des personnes spirituelles, mais non religieuses. 
nous faisons confiance au consensus des cliniciens, des autorités de santé publique, des chercheurs, des journalistes et du public en général, qui est favorable à la vaccination (Benin et coll., 2006; Streefland et coll., 1999; Tickner et coll., 2006). Bien entendu, la plupart des gens accorderaient également leur confiance aux ingénieurs civils, aux pilotes, aux électriciens, aux urgentologues et aux personnes chargées de veiller à la qualité de l'eau potable, pour ne nommer que quelques professions. Après tout, nous n'avons généralement ni le temps, ni les connaissances nécessaires pour évaluer les services que ces experts nous fournissent (et encore moins de les imiter). Si l'un d'entre nous dit avoir « confiance » en un pilote d'avion, par exemple, il l'affirme en supposant que celui-ci a suivi tous les cours de formation requis, comprend le fonctionnement de l'appareil et est prêt à gérer les situations d'urgence. La plupart d'entre nous, étant incapables d'évaluer rationnellement l'intégrité structurale des avions et les exigences de la formation des pilotes, présumons (généralement avec raison) que quelqu'un d'autre a veillé à ce que, dans ces deux cas, on respecte les normes établies par des autorités compétentes, un processus qui peut être concrétisé par l'imposition d'injonctions de la part d'un État qui veille au mieux à l'intérêt de ses citoyens.

La confiance se compare à une toile dans laquelle nous sommes tous imbriqués; certains d'entre nous sommes plus critiques quant à la solidité des liens, d'autres le sont moins et vont parfois jusqu'à s'en méfier (voir le chapitre 2 de O'Doherty, Smith et McMurtry et le chapitre 3 de Brunk dans le présent ouvrage, ainsi que Biss, 2014; Gardner, 2008). Même s'il est difficile d'imaginer un monde entièrement dépourvu de confiance - dans lequel personne ne voyagerait en avion, n'achèterait d'aliments en épicerie, ne monterait dans un ascenseur ni n'entrerait dans un immeuble -, il est évident que le lien de confiance peut être entamé ou même brisé en raison de la dépravation morale d'un individu (par exemple un enseignant faisant subir des abus sexuels à des enfants), d'une faute d'inattention (quand un avion s'écrase parce que le pilote n'a pas vérifié le niveau du carburant), d'une insensibilité (un médecin ne portant pas attention à un patient décrivant ses symptômes) ou de l'apparition de conséquences inattendues (médicament dont les effets cancérigènes apparaissent des années après son introduction).

Les vaccins sont produits par un système pluridisciplinaire complexe, où entrent en jeu cliniciens, universitaires, administrateurs, investisseurs privés, lobbyistes, personnel des soins de santé de première ligne, vérificateurs des effets indésirables, patients, décideurs politiques, leaders d'opinion, etc.; il est donc difficile pour un seul porte-parole de répondre aux trop nombreuses questions des réticents. Par conséquent, si les détracteurs de la vaccination qui s'appuient sur la religion ou la culture avancent parfois des arguments heuristiques erronés (Caulfield, 2015), il est aussi possible que certains partisans empruntent des raccourcis ou fassent valoir des arguments empiriques ou basés sur la confiance selon lesquels les vaccins ne sont qu'une autre méthode de s'assurer d'un bon niveau de vie. N'oublions pas, également, que dans ce cas-ci comme dans tous les autres où l'on invoque un argument largement accepté (pensons aux changements climatiques, à l'évolution, au traitement des eaux usées, au fluorure, etc.), on n'impose pas à tous les citoyens éduqués le devoir d'en vérifier soigneusement chaque volet; c'est d'ailleurs précisément à quoi sert ce qu'on appelle un consensus d'experts - soit un moyen nous permettant de vaquer à nos autres occupations pendant que des spécialistes gèrent d'autres décisions compliquées. 
Comme mes collègues auteurs le démontrent, des exemples fortement médiatisés d'incidents nuisant à la confiance - notamment la contamination de certains lots de vaccins contre la poliomyélite dans les premières années de la campagne d'éradication de cette maladie $^{14}$, l'étude de Tuskegee sur la syphilis ${ }^{15}$, le lien possible entre le syndrome de GuillainBarré et le vaccin contre la grippe ${ }^{16}$, la stratégie employée par la CIA pour localiser Oussama ben Laden et les preuves allant à l'encontre de déclarations de médecins niant toute influence des fabricants de médicaments dans le choix du traitement (Grande, Shea et Armstrong, 2011; Shnier et coll., 2013) - ont été mis au jour en même temps que se dessinait une nouvelle définition de la vérité, le tout ayant pour effet de créer d'importantes préoccupations quant à la fiabilité des vaccins et du système qui en assure la production et la promotion (Biss, 2014; Black et Rappuoli, 2010)17. Par ailleurs, depuis quelques années, on s'inquiète de plus en plus de la marchandisation, de l'instrumentalisation et de la sécurisation de la vie humaine, comme le démontre la présence massive, dans la culture populaire, de scénarios tels que ceux du Show Truman (1998), de La Matrice (1999), du Rapport minoritaire (2002), de la série Hunger Games (commencée en 2008) et d'Ex Machina (2015), ainsi que du concept de "transhumanisme » et de la notion apparentée de « singularité » avancés par Ray Kurzweil, selon qui l'intelligence artificielle pourrait enlever à l'homme la maîtrise du monde. Ces ouvrages, films et pensées relèvent probablement de la fiction, mais leur succès montre qu'il existe une angoisse générale à l'idée que les gouvernements ou les grandes sociétés - notamment celles du secteur des sciences et technologies - auraient intérêt à assurer leur domination sur les aspects privés et publics de la vie humaine, afin de protéger le néolibéralisme régnant ou de réprimer la dissidence. Ces produits culturels sont révélateurs de la crainte qu'éprouvent certains d'entre nous, imaginant un avenir dystopique pour une société où la science et la technologie sont de plus en plus incompréhensibles et où les entreprises acquièrent sur le monde un contrôle de plus en plus étendu. Les inquiétudes, notamment celles qui sont liées aux vaccins, ne peuvent donc être comprises que si l'on en fait l'attribut d'une société dans laquelle les citoyens et les détracteurs n'ont pas toute confiance que les médecins, les représentants du gouvernement et ceux qui tirent un bénéfice de la vente de vaccins accorderont la priorité à la santé des personnes et à la santé publique au détriment d'avantages politiques et économiques, feront

14. Voir les passages sur l'incident Cutter, au chapitre 9 du présent ouvrage, sous la plume de Monika Naus, Barbara Law et Aline Rinfret.

15. Il s'agit d'une étude dans le cadre de laquelle on n'a pas administré de pénicilline à des Afro-américains moins nantis atteints de syphilis, même si cela aurait été clairement le traitement efficace. On a plutôt permis à la maladie de suivre son cours naturel sur quarante ans (de 1939 à 1972), jusqu'à ce que l'on avertisse les autorités de ces violations à l'éthique. Quoique ce sombre épisode de l'histoire de la médecine ne comportait pas l'usage d'un vaccin, l'étude est un excellent symbole de l'instrumentalisation de la vie humaine et est souvent citée par les opposants pour inciter à la méfiance vis-à-vis des médecins qui prétendent protéger au mieux l'intérêt de leurs patients; certains chercheurs attribuent à cet incident un déclin d'intérêt pour la vaccination chez les Afro-américains (Moutsiakis et Chin, 2007).

16. Selon les Centers for Disease Control des États-Unis (CDC 2016) : «II est possible que le risque, pour le syndrome de Guillain-Barré (SGB), soit légèrement augmenté après la vaccination contre la grippe avec un vaccin inactivé. On l'estime à un ou deux cas supplémentaires par million de personnes vaccinées. C'est beaucoup moins que le risque de complications graves suivant une infection par la grippe, qui peut être prévenue par un vaccin. » Voir également « Guillain-Barré syndrome and Flu Vaccine », dernière modification le 16 octobre 2015, à l'adresse https://www.cdc.gov/flu/protect/vaccine/guillainbarre.htm.

17. Les partisans de la vaccination entonnent souvent le même refrain : il est vrai que des personnes ont souffert à cause des erreurs et des iniquités commises dans l'histoire récente de la médecine et de l'immunisation, mais la plupart (quoique non la totalité) des cliniciens en santé publique et des chercheurs en matière de vaccins ont agi de bonne foi; tout bien considéré, le nombre de personnes ayant été négativement touchées par ces incidents est négligeable, si l'on tient compte des millions de vies qui ont été protégées par des vaccins, ceux-ci étant soumis à un processus d'amélioration continu. 
immédiatement enquête sur les effets indésirables et compenseront suffisamment les (très rares) victimes des effets indésirables.

Nous en arrivons ainsi à une seconde crise, fortement liée à la première : la crise de la vérité. Dans les décennies ayant suivi la Deuxième Guerre mondiale - et surtout pendant les années 1960 -, les identités sexuelles alternatives, les formations politiques, les normes esthétiques et les approches en matière de rationalité ont gagné en crédibilité. À la fin de la Guerre froide, au moment même où naissait Internet, de nombreux obstacles à la communication et à l'épanouissement total de la réalité des classes subalternes ont été supprimés. Un certain éloignement du « positivisme » monolithique du Siècle des lumières, relativement à la nature de la vérité et de la connaissance, et l'adoption d'une approche accordant plus de mérites aux " autres modes d'acquisition du savoir » font partie des transformations culturelles profondes qui ont accompagné le féminisme, le multiculturalisme, le progrès scientifique (on mentionne souvent, quoique incorrectement, la théorie de la relativité d'Einstein), les conflits liés aux droits de la personne, la révolution sexuelle, la philosophie postmoderne et les rébellions postcoloniales, entre autres affluents de ce fleuve de libération. Notre société n'est pas un paradis égalitaire, point s'en faut - on trouve encore des hiérarchies et de la discrimination sur la base de la classe sociale, de la race, du sexe et de la religion et certains prétendent qu'Internet n'est qu'une nouvelle méthode de contrôle social et politique. Cependant, personne ne pourra nier qu'au cours des dernières décennies, les discours conventionnels associés à la science, à la politique, à la race, à la sexualité, à la religion et à l'identité personnelle ont été déstabilisés, en même temps que se produisait une prolifération de discours nouveaux et souvent libérateurs sur l'identité et la vérité.

Par conséquent, lorsqu'un étudiant affirme, comme si c'était une lapalissade, que tout est « relatif », qu'il y a de nombreuses «façons d'acquérir le savoir » et qu'une " prétention à la vérité » n'est que le reflet d'un programme politique favorisant un sexe, une région ou une classe sociale, il ne fait que reprendre certains des concepts fondamentaux issus des efforts d'émancipation nés à partir de 1970. Les lecteurs seront nombreux à appuyer les vastes changements entraînés par la remise en question, dans l'arène juridique, politique et sociale, de systèmes inflexibles et répressifs alliant puissance et vérité. Certains d'entre eux seront soulagés de voir la «science » et les grands fabricants de médicaments quelque peu malmenés par un public soupçonneux. Par contre, la mise en vedette des nouveaux modes d'acquisition du savoir a eu pour effet, peut-être inévitablement, de remettre en question les vaccins euxmêmes, ainsi que les méthodes logiques généralement associées à la science occidentale. C'est pourquoi on assiste aujourd'hui à une crise de la vérité. Ce n'est pas par accident que le Oxford English Dictionary a choisi « post-vérité » comme mot de l'année en 2016. Après tout, l'élection américaine de cette année-là a donné d'amples preuves que l'hyperbole et l'appel aux sentiments avaient beaucoup plus d'importance que la véridicité de base des allégations politiques ou scientifiques (sur des questions allant des changements climatiques à la science des vaccins, en passant par les déclarations publiques d'un candidat) ${ }^{18}$.

18. Voir la déclaration officielle des responsables de l'Oxford English Dictionary au sujet du choix de " postvérité » comme mot de l'année : "Word of the Year 2016 is ... ", le 8 novembre 2016, à l'adresse https:// en.oxforddictionaries.com/word-of-the-year/word-of-the-year-2016. Voir aussi Macdonald (2016) et Shellnutt (2016). 
Une telle approche, en matière de vérité, a diminué le prestige des sciences de la vie (et des autres formes d'activité sociale et individuelle); elle s'explique de diverses façons et a connu beaucoup de précurseurs (Bramadat et Kaufert, 2013). Ces changements ont eu de nombreux effets et ont notamment incité les Occidentaux à adopter une toute nouvelle approche à la santé et à l'interaction avec les professionnels de la santé (Coward et Ratanakul, 1999). Cette approche est inéluctablement liée à la présence grandissante d'Internet, ainsi qu'au relativisme et au subjectivisme qui caractérisent la vie culturelle contemporaine; de plus en plus de gens s'estiment en mesure de mieux s'informer sur leurs maladies, sur les protocoles de traitement et sur les interventions pharmacologiques. Comme l'explique Kata (2010) : "Entre 75 et 80 p. cent des utilisateurs (canadiens et américains) d'Internet recherchent en ligne des renseignements sur la santé. Parmi eux, 70 p. cent déclarent que ces renseignements ont une incidence sur leurs décisions en matière de traitement » (1709; v. Betsch et coll., 2012; Wolfe, Sharp et Lipsky, 2002). Nous observons que les patients se fient de plus en plus à Internet non seulement à titre de ressource dans le cadre d'une réflexion sur leur propre santé (et de leurs conversations avec les professionnels du domaine), mais aussi comme une source d'autodiagnostic toute-puissante et dépourvue d'obligations et de confirmation d'impressions préexistantes et d'attributs propres à la sous-culture; mais nous notons aussi qu'un abîme se creuse entre les anciennes et les nouvelles approches en matière de santé individuelle et de santé publique.

Une évolution culturelle de cette nature possède évidemment de nombreux avantages, car les groupes et les personnes qui professent un recours à d'autres modes d'acquisition du savoir se sentent moins isolés par ce qui leur semble un milieu médical autoritaire. Par contre, Internet, de par sa nature non réglementée, soulève des obstacles pour les professionnels de la santé, car on s'attend d'eux - peut-être pour la première fois - qu'ils collaborent avec des patients qui se sont fait de la médecine une idée fondée sur des inquiétudes, des demi-vérités, des rumeurs, un mercantilisme débridé et des théories du complot que véhicule Internet en même temps que des données et des théories scientifiquement admises (Scullard, Peacock et Davies, 2010). Poland, Jacobson et Ovsyannikova (2009) ont aussi observé ce qui suit : « Dans l'environnement actuel de communication de masse extrême et d'absence, pour les parents, de menaces immédiates de maladies contagieuses, une «bonne mère » fait ses devoirs et commence par s'intéresser aux effets indésirables des vaccins » (3241; v. Betsch et coll., 2012). Malheureusement, les parents accomplissent souvent ces devoirs sur une base individuelle et selon leurs propres dispositions, en plus de devoir composer avec une quantité incroyable de données probantes accessibles au public ainsi qu'avec la capacité des sous-cultures et cliques locales de contredire une information qui pourrait être considérée comme faisant autorité, ce qui rend difficile, pour chacun, la compréhension et l'évaluation des faits rapportés sur Internet. C'est pourquoi il est souvent vain de faire un effort pour en arriver à une conclusion raisonnable au sujet d'un problème de santé. Voici ce que l'on peut lire dans Healy et Pickering (2011) : 
«On ne doit jamais sous-estimer, également, l'importance d'une expérience ponctuelle, d'un clip sonore agrémenté d'une imagerie visuelle. Une vidéo de 30 secondes montrant un enfant dont la santé a prétendument été compromise à la suite de l'administration d'un vaccin exploite les pires craintes des parents et est plus efficace que celles qui décrivent la prévention ou l'élimination de maladies contagieuses dont ces parents n'ont jamais entendu parler et qu'ils n'ont même jamais vues. Ces impressions, jointes à des anecdotes que les parents auront entendues ou lues sur Internet, seront peut-être plus mémorables et les inciteront potentiellement à croire que les vaccins sont dangereux. » (129)

La capacité - maintenant décrite comme un droit - de tout être vivant dans une société démocratique libérale de refuser toute participation à un programme de vaccination parrainé par l'État (et réalisé avec des multinationales du médicament) fait partie de l'expansion de l'autonomie individuelle datant de la fin du siècle dernier. II n'est donc pas étonnant que les législateurs et les partisans de la vaccination se montrent très prudents avant de poser un geste ou d'adopter une politique pouvant limiter ce droit (Salmon et coll., 1999, 50; v. Streefland, 2001, 164). Pour preuve des soupçons avec lesquels ils doivent composer, voici une critique fort bien structurée des programmes de vaccination, figurant dans un mémoire adressé au ministère de la Santé de l'Ontario par un groupe d'opposants, le Vaccine Risk Awareness Network (maintenant appelé Vaccine Choice Canada) :

«Malgré les opinions conflictuelles, les erreurs médicales répétées et le recours abusif persistant à de nombreuses procédures médicales, l'attitude servile et l'appui inconditionnel que le public accorde aux médecins nous étonnent toujours. Mais cette situation n'est pas aussi surprenante qu'on le croit une fois que l'on a constaté la confiance similaire et injustifiée dont jouit le gouvernement, qui a le haut du pavé dans bien des aspects de notre vie, malgré toutes les erreurs qu'il a commises, de la mousse isolante d'urée-formaldéhyde aux radiographies pulmonaires obligatoires... comme nous croyons en la liberté humaine et en la capacité qu'a la vérité de se justifier par elle-même, comme disait Jefferson, nous acceptons le risque qu'il y ait, dans la société, quelques personnes irresponsables qui commettront de graves erreurs. II existe toutefois des personnes qui ne font pas autant confiance à autrui, qui ne croient pas vraiment à la liberté de l'homme et qui insistent pour prendre soin de nous, pour notre propre bien. En imposant leur « vérité »à tous, ils prennent un bien plus grand risque. En effet, si le gouvernement se trompe et nous oblige tous à agir de la manière prescrite par lui, cela nous oblige tous à nous tromper aussi. Un tel despotisme n'a plus sa place de nos jours (VRAN, 1984; v. aussi VRAN, 2005) ${ }^{19}$.

19. Le Vaccine Risk Awareness Network (maintenant appelé Vaccine Choice Canada) a publié le commentaire suivant au sujet de l'éclosion de rubéole à Woodstock, en mai 2005 :

"Depuis le début de mai, les médias, à l'incitation des autorités de santé publique, tentent de créer une panique au sujet de l'éclosion de rubéole qui est apparue, dans l'Ouest de l'Ontario, parmi les élèves d'une école chrétienne dont la plupart ne sont pas vaccinés. Les parents qui ont refusé de faire vacciner leurs enfants sont accusés de mettre les autres en danger; certains journaux et chaînes de nouvelles réclament même une politique de vaccination obligatoire pour tous... On s'attaque ainsi aux droits fondamentaux que la constitution canadienne accorde à chacun d'entre nous, c'est-à-dire la liberté de religion et de conscience et le droit, prévu dans la législation médicale canadienne, d'exercer un consentement éclairé pour toute procédure comportant un risque de blessure ou de décès. La vaccination compte parmi les procédures médicales! Malheureusement, ces accusations, ces attaques et l'appel à la suspension du droit fondamental de refuser une procédure médicale invasive proviennent d'un groupe de journalistes trop heureux de signaler des affaires sensationnelles, mais qui ne prennent pas le temps d'examiner tous les aspects du problème : la rubéole comme maladie, le vaccin RRO (soit celui qui contient le vaccin contre la rubéole), ses effets secondaires et son incidence sur l'épidémiologie de la maladie. (VRAN, 2005)

Se reporter également au site Web de Vaccine Choice Canada, consulté le 7 janvier 2017, à l'adresse http:// vaccinechoicecanada.com/. 
Les processus élémentaires que j'ai décrits plus haut sont succinctement saisis dans l'inquiétude sous-jacente qu'un État despote "imposerait » son opinion sur les "vérités » médicales à un public "servile » au sein duquel évoluent de nombreuses autres personnes plaidant en faveur d'une meilleure vérité, c'est-à-dire la leur. Imaginant peut-être déjà l'émergence de la « post-vérité » dans la société, Kata (2010) écrivait :

«Dans la société postmoderne, Internet, médium dominant, a libéré toute l'information autrefois captive. Vu le grand nombre de soi-disant experts qui y évoluent, l'opinion même du plus respecté des spécialistes de la vaccination n'est qu'une opinion parmi d'autres. Chacun peut, dans une certaine mesure, être considéré comme un expert. C'est pourquoi le recours à des autorités scientifiques ou médicales n'est pas aussi convaincant qu'autrefois. » (1715)

Comme le fait observer Paul Offit, auteur de Deadly Choices (2011), il est également important de noter que l'hésitation et l'opposition, en matière de vaccination, n'est pas uniquement le fait des personnes peu éduquées : "On trouve une réticence à la vaccination dans la classe moyenne supérieure, chez des parents diplômés d'universités et ayant fait des études plus avancées, qui probablement consultent Internet avant de prendre des décisions sur la santé et qui croient fermement qu'eux aussi, dans cette époque axée sur l'information, peuvent être des experts » (124; v. Akis et coll., 2011, 1171; Guay, Dubé et Laberge, chapitre 6 du présent ouvrage).

Les groupes actuels d'hésitants ont été précédés par d'autres, qui ont exprimé des doutes sur les programmes de vaccination dans le cadre desquels un gouvernement, collaborant avec des scientifiques, des fabricants de médicaments, des cliniciens et des établissements d'enseignement, s'est fixé comme priorité de vacciner une population entière contre une maladie donnée et a ainsi choisi d'imposer à tous un inconfort physique généralement léger et des effets indésirables en nombre négligeable (Guay et coll., 2009). Néanmoins, puisque la plupart de ces programmes relèvent d'une approche "descendante » en matière de santé et de science (v. Streefland, 2001, 160), ils risquent à coup sûr de promouvoir les craintes associées autant à la crise de vérité qu'à la crise de confiance. L'apparition et la croissance de mouvements critiquant la logique conventionnelle en général et les vaccins en particulier étaient prévisibles (v. Offit, 2011). On ne peut attribuer la responsabilité de ces deux crises (confiance et vérité) uniquement à un public trompé et laissé dans l'ignorance; le scepticisme (voire la paranoïa) qui ressort du discours des réticents à la vaccination est en partie attribuable aux erreurs des spécialistes de l'immunisation et, à l'occasion, à l'attitude des cliniciens, chercheurs, promoteurs de la santé publique et autres personnes participant au consensus scientifique entourant la vaccination. Bettinger et MacDonald (au chapitre 8) et Naus, Law et Rinfret (au chapitre 9) affirment que des erreurs tragiques ont eu un rôle à jouer dans la création du système qui est bénéfique pour bon nombre d'entre nous; il est plus difficile d'attribuer un résultat positif au paternalisme dont se plaignent régulièrement les critiques (v. Roberts et Mitchell, chapitre 10 du présent ouvrage; v. également Biss, 2014). Le partage de la responsabilité pour l'impasse actuelle ne constitue pas une solution au problème, mais il représente néanmoins un grand pas en avant. 


\section{Motifs religieux et culturels: ressemblances et dissemblances}

Après vous avoir brièvement décrit les tendances religieuses au Canada et celles qui, sur le plan plus large de la culture, pourraient amener la population à refuser la vaccination, j'aimerais remettre en question une hypothèse sur laquelle s'appuient ces deux sections et, en général, la controverse entourant les vaccins. II est souvent plus facile de faire une distinction analytique entre les motifs religieux et culturels de la réticence et de l'opposition à la vaccination; la plupart des auteurs de cet ouvrage procèdent d'ailleurs ainsi. Il est cependant essentiel de s'interroger non seulement sur les différences entre ces motifs, mais aussi sur ce qui les relie. Est-ce qu'on me permettrait de supposer que des forces sociales et psychologiques sont à l'œuvre au sein des sous-cultures qui se montrent hésitantes ou opposées à la vaccination pour des motifs culturels, par rapport à celles que nous disons agir pour des motifs religieux?

Dans cet ouvrage, nous nous intéressons à des groupes qui semblent unis par une pratique particulière (opposition ou réticence à la vaccination) dont l'origine réside dans plusieurs sous-cultures très distinctes; à cette ressemblance pourrait peut-être s'en ajouter une autre, à savoir que nombre d'entre eux ne sont pas d'accord ou se montrent méfiants en ce qui concerne le consensus scientifique prédominant, quoique non absolu, qui entoure la vaccination (en particulier) et la logique scientifique (en général). Parmi eux, on en trouve qui adhèrent à une explication non scientifique (sans données probantes) ou irrationnelle de la relation entre les maladies et les personnes ${ }^{20}$. Par manque d'espace, nous n'examinerons pas de près les différentes écoles de pensée qui s'intéressent à la définition de la science et de la méthode scientifique. Je fais donc appel à la définition conventionnelle en vertu de laquelle une explication scientifique (dans ce cas-ci, de l'innocuité et de l'efficacité des vaccins) peut mener à des hypothèses falsifiables, mais qui sont sujettes à révision, à rejet ou à confirmation après étude des données empiriques et au recours à des évaluations où l'on emploie des notions courantes à l'égard des données probantes et qui peuvent être répétées. Sur la base de cette définition générale et fonctionnelle de la science, Seth Mnookin (2012), auteur de The Panic Virus, a noté: «Je me suis aperçu que ce qui m'agaçait, c'était la présence constante d'une méthode de pensée (chez les sceptiques) qui va à l'encontre des principes du raisonnement par la déduction, qui est le fondement de la société rationnelle depuis le siècle des Lumières » (11; v. Offit, 2011). 
Certains d'entre nous cherchons à comprendre de quelle manière on pourrait repenser cette méthode que décrit Mnookin, de manière à pouvoir dialoguer plus efficacement avec ceux qui l'utilisent. Dans la sphère médicale comme dans le public, on continue de discuter des programmes d'immunisation contre le virus du papillome humain (VPH) (voir plus loin Roberts et Mitchell, auteurs du chapitre 10) et de la nécessité de la vaccination régulière contre la grippe, pour les adultes (voir Osterholm et coll., 2012). D'un autre côté, il s'est dégagé, chez ceux qui connaissent bien ce dossier (et comme d'autres coauteurs l'expliquent avec plus de précision), un solide consensus concernant l'importance et l'innocuité des vaccins contre la poliomyélite, la rougeole, la diphtérie et la rubéole. Malgré tout, comme Brunk, O’Doherty et d'autres auteurs l'indiquent, certaines des inquiétudes qu'éprouvent les gens à l'égard des vaccins découlent de préoccupations courantes, parfaitement justifiées et raisonnables; parmi les plus communes figurent le risque, les effets indésirables, la valeur des thérapies alternatives sur le plan médical et l'influence des grandes sociétés pharmaceutiques. Ce qui intéresse Mnookin - ainsi que les membres de notre équipe de recherche -, c'est que la réticence et l'opposition demeurent malgré le fait que la plupart de ces préoccupations rationnelles aient obtenu réponse de la part des épidémiologistes, des spécialistes des maladies contagieuses, des représentants de santé publique et des cliniciens. Pour comprendre la persistance de telles profondes inquiétudes au sein de nombreuses sous-cultures de notre société malgré les réponses obtenues, nous avons besoin d'explications supplémentaires qui tracent un portrait plus précis de ces convictions inébranlables. Je propose l'idée que, dans la plupart des sous-cultures opposées à la vaccination, on entretient des doutes qui sont ancrés dans ce que l'on appelle en général un mode de réflexion ou des expériences spirituelles, magiques ou irrationnelles. On remarquera que ce mode et ces expériences font partie des principaux éléments de la vie religieuse, chez la plupart des gens ${ }^{21}$.

Réfléchissons sur l'analogie que l'on pourrait établir entre les sensibilités religieuses implicites et expresses et les discours anti-vaccination et considérons l'un des exemples les plus connus d'une objection issue non de la religion, mais de la culture. En 1998, dans un article publié dans The Lancet, Andrew Wakefield a émis l'opinion que le vaccin contre la rougeole, la rubéole et les oreillons (RRO) pouvait avoir un rôle à jouer dans l'émergence de l'autisme et cette affirmation est devenue l'un des plus importants piliers des sous-cultures réticentes ou opposées à la vaccination (Gerber et Offit, 2009). L'allégation était exprimée dans un langage scientifique généralement incompréhensible pour tous sauf les spécialistes, mais, malgré tout, l'article a donné une validation scientifique à l'argument, au départ non scientifique, avancé par d'autres moyens - notamment, à l'occasion, par des parents éperdus disant avoir observé des symptômes de l'autisme peu de temps après la vaccination (Kata 2010, 1713). D'autres médias et ex-leaders de la médecine se sont joints aux sous-cultures

21. Certaines personnes fondent leur vie religieuse sur un respect consciencieux et systématique des systèmes théologiques et doctrinaux. Mon but est de rappeler que ceux-ci ne sont que l'une des dimensions de cette vie et pas nécessairement la plus importante. Depuis ses débuts, l'histoire religieuse, pour l'essentiel, relate la vie, les pensées, les expériences et les aspirations d'une élite intellectuelle, religieuse et sociale (composée presque entièrement d'hommes). Par contre, des descriptions et évaluations récentes de vies religieuses ont souvent mis l'accent sur ce qui peut être dit au sujet des expériences de gens ne faisant pas partie de l'élite. On y laisse entrevoir que les dimensions supra-rationnelles, émotionnelles et subjectives de la religion, quel que soit le nom qu'on leur donne (acte de foi, rencontre du surnaturel, relation personnelle avec un dieu, sens d'un mystère ineffable, confiance dans des pouvoirs qui semblent réels, mais ne sont pas scientifiquement validés), sont les caractéristiques les plus typiques et attirantes de la religiosité, pour la plupart des gens, en toute probabilité. Je fais ici allusion à l'importance que l'on donne dans certains milieux universitaires à la « religion vécue », qui met en vedette les sensibilités religieuses souvent syncrétiques des croyants ordinaires, plutôt que la structure intellectuelle ou théologique associée à l'élite d'une population religieuse donnée (voir Hall, 1997; Orsi, 2004). 
opposées à la vaccination (Mnookin, 2012; Offit, 2011), s'ils n'en étaient pas déjà membres, en faisant de la recherche de Wakefield un des principaux piliers de leur argumentation. Cependant, quelques années après la publication, on a découvert que les méthodes, l'intégrité et les conclusions de Wakefield étaient fallacieuses et, en 2010, The Lancet a pris une mesure très inhabituelle en retirant cet article de ses pages. ${ }^{22}$ Par là-dessus, le permis d'exercice de la médecine de Wakefield fut révoqué en raison de ses gestes frauduleux et contraires à l'éthique. Vu le mérite que bien des gens ont accordé à ses arguments, on aurait cru que cet événement aurait entraîné la disparition de la sous-culture accusant le vaccin RRO d'être lié à l'autisme. La théorie de Wakefled avait cependant fortement entamé la confiance du public à l'égard de la vaccination et les groupes ou l'ensemble des sous-cultures auxquels elle donnait une validation ne sont pas disparus. Wakefield fut plutôt, pour beaucoup, considéré comme un martyr (Dominus, 2011; Kata, 2010, 1713) et le plaidoyer dressé contre lui et ses premières (et peu fiables) recherches sur le lien entre le RRO et l'autisme fut présenté comme un autre exemple des milieux médicaux protégeant la doctrine orthodoxe bien établie en médecine et en pharmacologie (MacDonald, 2007; v. Poltorak et coll., 2005) ${ }^{23}$.

II faut aussi porter attention à la puissance des sensibilités irrationnelles dans une sousculture de la médecine alternative qui est étroitement liée à l'opposition et à la réticence à la vaccination. L'homéopathie est née à la fin du XVIII siècle et est devenue, depuis quelques dizaines d'années, un élément important du mouvement en faveur d'une médecine alternative (ou complémentaire) (Benin et coll., 2006; Ernst, 2002; Zuzak et coll., 2008). Elle prescrit l'absorption de substances qui sont diluées à un point tel que, dans certains cas, pas même une seule molécule de la substance originale ne peut être détectée (Ernst, 2005); les contenants où elles sont versées sont ensuite projetés contre une surface (ce que l'on appelle la «succussion ») pour en accroître la puissance. En vertu du principe voulant que «le semblable guérit son semblable » et de la notion que la solution contient encore quelques propriétés curatives (voir Dubé, Sauvageau et Gagnon, au chapitre 7 du présent ouvrage), les patients d'un homéopathe absorbent de petites quantités de substances diluées qui, à leur teneur originale, causeraient les symptômes qu'éprouve le patient. Comme l'expliquent Benin et coll., (2006), Ernst (2002), Zuzak et coll., (2008) et Dubé, Sauvageau et Gagnon (chapitre 7 du présent ouvrage), la corrélation entre l'hésitation face à la vaccination et l'engagement en faveur de l'homéopathie est forte - principalement parce que les homéopathes et leurs patients croient que leurs remèdes « naturels » suffiront à les protéger des infections ou leur permettront de composer avec les symptômes s'ils tombent malades.

22. Voir la déclaration des rédacteurs du Lancet (2010). On notera qu'en 2011, Wakefield occupait le premier rang de la liste des pires médecins de Medscape; se reporter à " Physicians of the Year: Best and Worst ", Medscape, 2011, consulté le 7 janvier 2017, à l'adresse http://www.medscape.com/features/slideshow/physiciansof-the-year/2011. En janvier 2012, la revue TIME Magazine a classé Wakefield dans sa liste des plus grands falsificateurs scientifiques; voir Alice Park, " Great Science Frauds », TIME Magazine, 12 janvier 2012, à l'adresse http://healthland.time.com/2012/01/13/great-science-frauds/slide/andrew-wakefield/.

23. Comme l'ont noté Poland, Jacobson et Ovsyannikova (2009) : "Le lobby des opposants radicaux à la vaccination est devenu une " arme de distraction massive » à cause de ses activités d'information du public et des législateurs au sujet des risques et des bienfaits des vaccins » (3241). Les hypothèses de Wakefield reçoivent encore un appui, comme on peut le voir en lisant le blogue de Barbara Loe Fisher, sous le titre « Doctors, Judges, and Juries Hanging Their Own » (2010), à l'adresse http://vaccineawakening.blogspot.ca/2010/01/ vaccines-doctor-judges-juries-hanging.html. Fisher préside le National Vaccine Information Centre et elle a écrit en 1985 l'ouvrage «A Shot in the Dark», dont l'influence a été grande. 
Les homéopathes et leurs patients ont reçu une bien mauvaise nouvelle en 2005, quand une méta-étude portant sur les évaluations empiriques de l'homéopathie, publiée dans The Lancet, a démontré l'absence d'effet positif (sans utilisation de placebo) de la thérapie (Shang et coll., 2005). Cependant, comme le font remarquer Dubé, Sauvageau et Gagnon (chapitre 7) et comme on peut le voir par une simple visite d'Internet ou en écoutant d'une manière attentive ce qui se dit au bureau ou chez ses voisins, le public s'intéresse toujours à l'homéopathie comme traitement complémentaire légitime ${ }^{24}$.

Il est bon de noter que ces deux articles publiés dans The Lancet ont été fortement médiatisés - et leur jargon scientifique rendu assez compréhensible pour les profanes - par des sources d'information grand public de bonne renommée (y compris Radio-Canada, la BBC, le Globe and Mail et le New York Times, pour n'en nommer que quelques-unes). Signalons que Wakefield, les homéopathes et leurs partisans ont bien fait connaître leur position et continuent de le faire ${ }^{25}$. On ne peut qu'être intrigué par le fait que des preuves empiriques tout à fait convaincantes n'ont pas réussi à mettre au ban de l'histoire ni l'homéopathie, ni l'allégation du lien entre le vaccin RRO et l'autisme (Hobson-West, 2003).

Heureusement, les spécialistes de la religion s'intéressent à des phénomènes semblables depuis de nombreuses années. En 1956, Leon Festinger, H.W. Riecken et S.W. Schachter ont publié When Prophecy Fails: A Social and Psychological Study of a Modern Group that Predicted the End of the World, qui fut le premier d'une ample documentation (v. Dawson, 1999; Dein, 2001; Tumminia, 1998) susceptible de jeter un certain éclairage sur les processus à l'œuvre dans la réticence et l'opposition à la vaccination. Que se passera-t-il, se demandent ces sociologues, si la fin du monde ne se produit pas un jour donné ou si le Messie n'arrive pas au moment indiqué par les prophètes? Festinger, Riecken et Schachter (1956) se sont intéressés aux manières qu'utilisent les groupes religieux et les fidèles pour réduire la « dissonance cognitive » au moment où leurs allégations et leurs espoirs sont désavoués par les faits. Ces auteurs ont indiqué que certains membres pourraient, à cette fin, augmenter encore leur engagement personnel dans les groupes potentiellement mis en danger et même accroître leurs activités de prosélytisme; d'autres analystes (Dawson, 1999; Snow et Machalek, 1982) ont cependant par la suite fait une mise en garde, car nous surestimons souvent les crises que de telles données empiriques contradictoires causent chez les membres ordinaires. En fait, les personnes réunies dans ces sous-cultures pourraient fort bien ne pas être aussi troublées par des preuves contraires que ne le supposent (ou souhaitent) les universitaires, les promoteurs de santé publique et les médecins. Pour peu qu'il remarque de telles informations ou preuves troublantes, l'initié les range parmi les données non pertinentes ou celles qui représentent les intérêts d'un groupe dominant préoccupé de conserver sa place ou - comme l'indique l'anecdote du travailleur de santé publique (parent d'un enfant gravement autiste; se reporter au chapitre 3, sous la plume de Brunk) - ne peut, ni ne veut s'en servir pour modifier ses valeurs, remédier à son angoisse ou prendre un autre engagement émotionnel. Détromper

24. Les remèdes homéopathiques sont en effet offerts en Amérique du Nord non seulement dans les magasins d'aliments naturels, mais aussi dans les grandes pharmacies, y compris celle de l'Université de Victoria. Voir Bodeker et coll. (2005) et "Register a Homeopathic Medicine or Remedy », GOV.UK, dernière modification le 7 décembre 2016, à l'adresse https://www.gov.uk/guidance/register-a-homeopathic-medicine-or-remedy.

25. L'homéopathie a en effet bénéficié de nombreux défenseurs, mais une longue discussion sur ce sujet ne relève pas du présent ouvrage. Se reporter à Caulfield (2005), Fisher (2006), Kudha-Bukhsh (2003) et Riley et coll., (2001), ainsi que « Homeopathy: New Evidence », Medical News Today, 14 novembre 2008, à l'adresse http:// www.medicalnewstoday.com/releases/ 129436.php. 
une personne est souvent inefficace, car l'on suppose généralement qu'il suffira de contester des allégations erronées (parfois en les ridiculisant) et de transmettre une information précise.

Une étude réalisée par Snow et Machalek, en 1982, au sujet de la « mise en suspension de l'incrédulité » qui serait, paraît-il, évidente quand un individu plonge dans un mouvement religieux, surtout si les "structures de plausibilité » de ces traditions sont menacées, fait entrevoir ce que beaucoup considèrent encore comme une conclusion difficile à croire, à savoir qu'il relèverait d'une certaine vanité intellectuelle de prétendre que l'incrédulité et le doute sont les attitudes standard du cerveau humain et qu'il faudrait les mettre de côté pour s'intégrer dans un mouvement culturel ou religieux atypique ou accepter des allégations qui se trouvent sans validation empirique. En fait, bien rares sont les personnes qui envisagent le monde dans un esprit toujours sceptique, voire analytique. La plupart d'entre nous, loin d'avoir à abandonner nos approches naturellement ironiques ou sceptiques pour accepter une nouvelle conviction (quelle qu'elle soit) et loin de baser notre décision sur les allégations médicales à accepter ou à refuser une analyse des données, théories et hypothèses faite de sang-froid, nous nous fions plutôt sur des raccourcis intellectuels souvent fautifs ou sur l'opinion des autres dans notre évaluation des preuves. Autrement dit, nous avons en général à mettre de côté une quelconque conviction ou valeur pour adopter une attitude dubitative ou une autre forme de conviction (v. Haidt, 2012; Latour, 2009). En fait, il serait plus exact de dire que chacun va souvent du rationnel à l'irrationnel, en termes d'explications ou de visions du monde, pour faire la lumière non seulement sur des expériences personnelles complexes, mais aussi des enjeux scientifiques apparemment simples. Prenez l'exemple du nombre de chrétiens américains qui croient aux principes de base du christianisme, mais aussi à la réincarnation (22 p. cent) ${ }^{26}$, du nombre de personnes qui croient que la Terre est jeune (c'està-dire qu'elle existe depuis moins de dix mille ans) et qu'il y a des anges et des démons, mais en même temps accordent de l'importance aux démarches scientifiques en soins de santé et en ingénierie de base (Bramadat, 2000), des personnes qui croient qu'il y a une raison derrière toute chose (Thagard, 2010), tout en reniant les perspectives mondiales d'origine religieuse et des personnes qui croient aux « remèdes de grand-mère » autant qu'à la biomédecine (Moore et McClean, 2010). Non seulement le passage d'une perspective du monde à une autre est-il la règle plutôt que l'exception, mais ce fait ne semble que rarement susciter de crises existentielles. La vérité, du moins selon la définition généralement étroite qu'en donne un certain consensus de libéraux intellectuels et scientifiques, n'est tout simplement pas si importante qu'on pourrait l'imaginer (ou le préférer). Le fait que bon nombre d'entre nous en soyons étonnés est bizarre en soi, en raison de la multiplication des preuves du rôle important joué par les motifs prérationnels, irrationnels, inchoatifs et contradictoires dans nos convictions et décisions (Ariely, 2008; Bramadat, 2000; Gardner, 2008; Haidt, 2001; Haidt, 2012; Hall, 1997; Hobson-West, 2003; Kata, 2010; Spier, 2001) et du fait que nous vivons dans une société de la «post-vérité ».

26. Voir «Many Americans Mix Multiple Faiths`», Pew Research Center, 9º́ecembre`2009, à l'adresse http:// www.pewforum.org/Other-Beliefs-and-Practices/Many-Americans-Mix-Multiple-Faiths.aspx\#3. 
Les partisans de la vaccination se basent sur des données plus récentes pour promouvoir leur point de vue et cela témoigne d'un ensemble de convictions tout à fait légitimes au sujet d'une forme particulière de raisonnement scientifique et de la confiance que ce dernier inspire (Dubé et coll., 2013; Hobson-West, 2003; Kata, 2010, 2012). En revanche, l'irritation que ressentent certains cliniciens, scientifiques et professionnels de santé publique à l'endroit des réticents et opposants à la vaccination leur est inspirée parce qu'ils surestiment le pouvoir d'un raisonnement particulièrement étriqué et sous-estiment celui des aspects autres que cognitifs de la religiosité, voire de toute conviction fortement ancrée. Il est difficile et peut-être futile de s'attendre à ce que le rationalisme scientifique puisse convaincre la plupart des gens religieux de modifier leur opinion et leurs sentiments à l'égard de leurs sensibilités religieuses les plus sincèrement ressenties (et jusqu'ici tous ces efforts se sont soldés par un échec), car l'aspect rationnel de la vie de la plupart des gens religieux n'est qu'une dimension parmi d'autres et n'est souvent pas la plus importante. Cela ne signifie pas que les gens religieux ont une âme enfantine ou sont des ignorants; cela veut simplement dire que de critiquer les arguments qu'ils avancent ou de dénigrer les opposants à la vaccination sur la seule base de la raison et des données probantes n'engendreront pas, chez eux, le genre de crise existentielle ou intellectuelle auquel on s'attend ${ }^{27}$.

Comment les partisans de la vaccination et les cliniciens peuvent-ils intégrer cet argument dans leurs dialogues quotidiens avec les réticents et les opposants à la vaccination? Et si la justification rationnelle exprimée par une personne ou une collectivité pour expliquer sa position en matière de vaccination était en fait l'expression d'autres forces irrationnelles ou supra-rationnelles? Parmi les réponses possibles, ne pourrait-on pas dire que, si une sousculture croit qu'un vaccin en particulier est à l'origine du syndrome de mort subite du nourrisson (SMSN), il faut absolument repérer les discours sociaux (Kata, 2010, 1715) ayant servi à définir et lier le groupe. En plus, il est important de prendre au sérieux les préjugés des individus et des groupes à l'égard des vaccins, des professionnels de la santé, du capitalisme et de la science (entre autres sujets d'inquiétude). Si un de ceux-ci est lié non pas principalement par une allégation scientifique particulière et vérifiable sur le SMSN, mais bien par un engagement profond à l'égard de l'homéopathie, par une aversion viscérale relativement à la médicalisation de la naissance, par l'expérience émotionnelle dévastatrice associée à la mort subite d'un nouveau-né, par la croyance (quand même assez répandue) que rien n'arrive sans raison ou, peut-être, par le marketing rusé d'un leader charismatique au sein d'un mouvement de santé alternative, les partisans de la vaccination devront porter une attention particulière aux forces complexes de l'irrationalité qui sont à l'œuvre (v. Healy et Pickering, 2011; Gardner, 2008). Quand un groupe est influencé par autant de forces, il ne suffira pas, pour apaiser ses inquiétudes fondamentales, de simplement lui présenter ce que les scientifiques considèrent comme étant des preuves définitives que le lien entre le vaccin et le SMSN n'est pas appuyé par des données médicales probantes. Comme le suggèrent d'autres auteurs (voir le chapitre 11, sous la plume de François $D$. Boucher), la meilleure réponse clinique à une réticence et une opposition à la vaccination profondément ancrées dans un ensemble de motifs consiste à s'efforcer de nouer une relation sûre et de confiance avec le patient et à discuter franchement de toutes les variables pouvant s'immiscer dans une interaction clinique de longue durée. Certains cliniciens trouveront cette tâche difficile, mais celle-ci semble être un des meilleurs

27. Dans les écrits de certains des «nouveaux athées ${ }^{\circ}$ ", tels que Christopher Hitchens, Richard Dawkins et Sam Harris, on distingue une profonde consternation car, après qu'on leur a exposé une logique et des données, la plupart des gens persistent à croire, à se réunir et à appuyer des idées, des pratiques, des sensibilités morales et, bien souvent, les institutions associées à la religion. 
moyens de s'assurer que le patient poursuit le dialogue sur les interventions préventives importantes (Benin et coll., 2006; Brownlie et Howson, 2005; Leask et coll., 2012; Omer et coll., 2009).

La principale question sur laquelle porte cechapitreest la suivante:comment pouvons-nous expliquer la manière qu'utilisent les personnes et les groupes (c'est-à-dire les sous-cultures) pour interpréter ce qui semble une évidence scientifique relativement crédible, mais qui va à l'encontre de leurs croyances et de leurs instincts de base? On doit certainement trouver une explication pour la survivance de ces théories démenties dans l'inquiétude généralisée qu'éprouvent les parents pour le bien-être de leurs enfants au moment même où tout notre environnement semble être en péril; on pourrait également mettre en cause l'information erronée que véhicule Internet et peut-être aussi la méfiance qu'éprouvent ces mouvements relativement à des expériences négatives qu'auraient subi quelques personnes auprès de médecins. Néanmoins, le fait que des données probantes clairement présentées, sous des angles différents, par des journalistes, des universitaires, des médecins et des défenseurs de la santé publique n'aient guère eu d'effet sur les personnes et les mouvements visés - ceux-ci étant en même en progression, selon les derniers rapports-donnent à penser que la prudence s'impose, pour ne pas surestimer l'importance que les gens accordent au raisonnement conventionnel basé sur ce type de données. Je suggère que l'on consulte des anthropologues, des philosophes, des historiens, des psychologues et des spécialistes des sciences religieuses pour en savoir plus sur les moyens que prennent les gens pour conserver des convictions profondes, des styles de vie, des rituels et des habitudes qui ne sont guère affectés par des données scientifiques ordinaires - ou avec lesquelles ils interagissent de façon très créative. Le fait de mettre en vedette les instruments heuristiques sur lesquels comptent les partisans de la vaccination dans leur vie personnelle et professionnelle pourrait en réalité améliorer leur attitude envers les critiques de la vaccination et peut-être même dissiper l'impasse à laquelle aboutissent souvent les relations entre les deux parties. Les sociologues pourraient également montrer les similarités entre les opposants à la vaccination et les groupes religieux, la plupart n'étant pas fondées sur les données probantes, dans leur définition la plus étroite. Enfin, les experts en sciences religieuses pourraient illustrer comment des opposants manifestant des points de vue apparemment radicalement différents (p. ex. les chrétiens fondamentalistes, musulmans non orthodoxes et femmes athées spécialistes de l'étude des religions) pourraient malgré tout entamer des discussions fructueuses au sujet d'enjeux religieux et moraux d'un intérêt mutuel. De telles activités constructives sont possibles - du moins en théorie - dans les universités et ailleurs dans la société civile et on pourrait s'en servir pour créer un modèle pour le maintien de relations entre les cliniciens et les représentants de santé publique, d'une part, et les patients et parents réticents et opposés à la vaccination, d'autre part.

En ce qui concerne les études de cas visant le lien RRO-autisme et l'homéopathie, mon impression est que nous assistons non pas à un simple scepticisme vis-à-vis de la médecine conventionnelle - une interrogation qui serait plus à sa place dans les milieux cliniques soumis à la méthode scientifique -, mais à une convergence des crises de vérité et de confiance, accompagnée de la popularité croissante d'approches différentes, supra-rationnelles au corps humain, à la science et à la vérité qui déstabilisent des autorités médicales. Les questions que l'on se pose actuellement sur différentes pratiques médicales (ycompris l'immunisation) et, plus généralement, sur le raisonnement scientifique basé sur les données probantes témoignent d'une société dans laquelle les citoyens ont acquis, plus que jamais, une liberté de chercher par eux-mêmes leurs vérités personnelles, spirituelles et scientifiques et nourrir leur propre 
sens de l'authenticité (Taylor 1992). Les interrogations sur les approches conventionnelles en matière de données probantes, de compétence des autorités et de raisonnement, qui forment le noyau des arguments religieux et culturels des réticents et des opposants à la vaccination, ont trouvé un terrain fertile dans les changements sociaux relativement récents qui sont associés à ces réalisations.

\section{Conclusion}

En temps ordinaire, le passage du scientifique au religieux, en matière d'explications, a des incidences salutaires sur le plan psychologique, mais très limitées sur le plan public. Une telle souplesse permet à chacun de recourir aux discours distinctifs de la science, de la technologie, de la religion ou de la spiritualité pour s'adapter aux changements souvent impromptus de son existence. Par contre, si l'on accepte les arguments présentés par Naus, Bettinger, MacDonald et d'autres auteurs du présent ouvrage, la réticence et l'opposition à la vaccination ne sont pas seulement des exemples d'une oscillation sans danger et personnellement utile entre une perspective scientifique et une perspective supra-rationnelle de la vie humaine. Certes, le déclin graduel de la protection des personnes et des populations à la suite de la popularité croissante de la réticence et de l'opposition à la vaccination est un problème important - et dès lors d'ordre politique - en matière de santé publique (De Serres et coll., 2013; Oostvogel et coll., 1994; Omer et coll., 2009; Spier, 2001).

Les forces sociales complexes dont j'ai tracé le portrait dans le présent chapitre et dans l'introduction - notamment l'hétérogénéité des groupes, l'influence d'une certaine forme de relativisme, la remise en cause de l'autorité de la science et de la médecine, la prolifération de sites Web de diagnostic et de soutien scientifiques et pseudo-scientifiques, l'autonomie croissante des patients et la nature quasi religieuse ou supra-rationnelle des arguments critiques de la vaccination, ont, réunies, rendu difficile la détermination de la nature et de la portée du problème et le choix des meilleures mesures pour y remédier (Mnookin, 2012; Kata, 2010). Les partisans de la vaccination sont nombreux à se demander si une " tempête parfaite » ne se prépare pas dans le domaine de la santé publique; ses effets pourraient être dévastateurs sur les citoyens dont les systèmes immunitaires ne sont pas encore développés (les nourrissons) et qui sont mis en danger à cause de l'âge, d'un traitement médical ou du fait qu'un ou plusieurs vaccins destinés aux enfants ne leur ont pas conféré l'immunité recherchée. La situation est difficile, mais sa complexité en renforce encore l'urgence.

Même quand un périodique laïque rapporte des incidents qui ne semblent pas avoir de relation claire avec des institutions ou des traditions religieuses officielles, la rhétorique religieuse est plutôt souvent intégrée à la discussion. Par exemple, dans un article du New York Times Magazine publié en 2011 qui a fait date, la journaliste Susan Dominus décrivait la fin de la carrière de Wakefield en mentionnant l'apocalypse qui a suivi, son statut de gourou et son sens de la mission. Elle ajoutait que ses partisans endossaient entièrement sa théorie et faisaient de lui un martyr. Selon elle, pour les groupes opposés à la vaccination, Wakefield personnifiait à la fois Nelson Mandela et Jésus-Christ. Elle ajoutait aussi que cet homme était un leader religieux dont la foi ne pouvait être mise en doute, mais qui utilisait parfois quelques trucs de magie pour stimuler celle de ses fidèles et concluait en observant qu'on peut croire pratiquement n'importe quoi, à la condition d'y mettre un peu du sien. 
Bien que la religion, dans son sens institutionnel étroit, n'ait pas figuré comme facteur important dans la recherche de Wakefield, dans sa popularité ou dans les reproches que lui ont fait les scientifiques et les journalistes, il faut presque, pour expliquer tout ce scandale, faire appel aux explications et métaphores religieuses décrites ci-dessus. Cela ne constitue pas simplement un rappel des origines religieuses de la culture nord-américaine; c'est aussi une indication que les moyens pris par les partisans de Wakefield pour contester les preuves scientifiques ressemblaient, à bien des points de vue, à ce à quoi nous devons nous attendre, selon les sociologues, de la part de gens profondément et sincèrement religieux qui doivent composer avec des preuves contradictoires. La distinction entre les différentes formes de réticence et d'opposition fondées sur la religion et la culture peut être profitable sur le plan de l'analyse, mais elle pourrait aussi empêcher un examen de quelques points communs grâce auxquels nous serions bien plus en mesure de comprendre les troublantes interrogations actuelles au sujet des vaccins. 


\section{BIBLIOGRAPHIE}

Akis, S., S. Velipasaoglu, A.D. Camurdan, U. Beyazova, and F. Sahin. 2011. "Factors Associated with Parental Acceptance and Refusal of Pandemic Influenza A/H1N1 Vaccine in Turkey." European Journal of Pediatrics 170 (9): 1165-72. http://dx.doi. org/10.1007/s00431-011-1425-6.

Alphonso, C. 2012. "Whooping Cough Makes Deadly Return across Canada." Globe and Mail, 23 July. http://www.theglobeandmail.com/life/health-and-fitness/health/whooping-cough-makes-deadly-return-across-canada/article4436946/.

Ariely, D. 2008. Predictably Irrational: The Hidden Forces that Shape Our Decisions. New York: Harper Collins.

Arnal, W.E. 2000. "Definition." In Guide to the Study of Religion, edited by W. Braun and R. McCutcheon, 21-34. London: Cassell.

Basrur, S. 2005. Annual Report of the Chief Medical Officer of Health to the Ontario Legislative Assembly. Toronto: Ministry of Health and Long-Term Care.

Beaman, L., and P. Beyer. 2008. Religion and Diversity in Canada. Leiden: Brill. http://dx.doi.org/10.1163/ ej.9789004170155.i-228.

Benin, A.L., D.J. Wisler-Scher, E. Colson, E.D. Shapiro, and E.S. Holmboe. 2006. "Qualitative Analysis of Mothers' Decision-Making about Vaccines for Infants: The Importance of Trust." Pediatrics 117 (5): 1532-41. http://dx.doi.org/10.1542/peds.2005-1728.

Betsch, C., N.T. Brewer, P. Brocard, P. Davies, W. Gaissmaier, N. Haase, Julie Leask, Frank Renkewitz, Britta Renner, Valerie F. Reyna, et al. 2012. "Opportunities and Challenges of Web 2.0 for Vaccination Decisions." Vaccine 30 (25): 3727-33. http://dx.doi.org/10.1016/j.vaccine.2012.02.025.

Bibby, R. 1987. Fragmented Gods: The Poverty and Potential of Religion. Toronto: Irwin.

- 1993. Unknown Gods: The Ongoing Story of Religion in Canada. Toronto: Stoddart.

- 2011. Beyond the Gods and Back. Lethbridge: Project Canada.

Biss, Eula. 2014. On Immunity: An Innoculation. Minneapolis: Graywolf Press.

Black, S., and R. Rappuoli. 2010. "A Crisis of Public Confidence in Vaccines." Science Translational Medicine 2 (61): 61mr1. http://dx.doi.org/10.1126/scitranslmed.3001738.

Bodeker, G., C.K. Ong, C. Grundy, G. Burford, and K. Shein. 2005. WHO Global Atlas of Traditional, Complementary and Alternative Medicine. Kobe: WHO Centre for Health Development.

Bramadat, P. 2000. The Church on the World's Turf: An Evangelical Student Group at a Secular University. New York: Oxford University Press.

Bramadat, P., and J. Kaufert. 2013. "Religion, Spirituality, Medical Education, and Hospice Palliative Care." In Spirituality in Hospice Palliative Care, edited by P. Bramadat, K.I. Stajduhar, and H. Coward, 60-85. Albany: SUNY Press.

Bramadat, P., and D. Seljak, eds. 2005. Religion and Ethnicity in Canada. Toronto: Pearson

- eds. 2008. Christianity and Ethnicity in Canada. Toronto: University of Toronto Press. http://dx.doi.org/10.3138/9781442687622.

- 2013. "Between Secularism and Post-Secularism: A Canadian Interregnum." In The Secular State and Religious Diversity: Secularism, Tolerance, and Accommodation, edited by A. Laliberté, B. Berman, and R. Bhargava, 97-119. Vancouver: UBC Press. 
Brownlie, J., and A. Howson. 2005. "'Leaps of Faith' and MMR: An Empirical Study of Trust." Sociology 39 (2): 221-39. http://dx.doi.org/10.1177/0038038505050536.

Casanova, José. 1994. Public Religion in the Modern World. Chicago: University of Chicago Press.

Caulfield, T. 2005. "A Systematic Review of how Homeopathy is Represented in Conventional and CAM Peer Reviewed Journals." BMC Complementary and Alternative Medicine 5: 5-12.

- 2015. Is Gwyneth Paltrow Wrong About Everything? When Celebrity Culture and Science Clash. Toronto: Viking.

Centers for Disease Control and Prevention (CDC). 1999. "Achievements in Public Health, 1900-1999: Impact of Vaccines Universally Recommended for Children - United States, 1900-1998." Morbidity and Mortality Weekly Report (MMWR) 48 (12): 243-8.

- 2016. "Possible Side-Effects from Vaccines." Centers for Disease Control and Prevention. Last modified 2 December. https://www.cdc.gov/vaccines/vac-gen/sideeffects.htm.

Chandler, S. 2008. "The Social Ethic of Religiously Unaffiliated Spirituality." Religion Compass 2:1-17.

- 2010. "Private Religion in the Public Sphere: Inner Life Spirituality in Civil Society." In Religions of Modernity: Relocating the Sacred to the Self and the Digital, edited by S. Aupers and D. Houtman, 69-88. Leiden: Brill. http://dx.doi.org/10.1163/ ej.9789004184510.i-273.28.

College of Physicians of Philadelphia. 2017. Cultural Perspectives on Vaccination. The History of Vaccines. Accessed 7 January 2017. http://www.historyofvaccines.org/ content/articles/cultural-perspectives-vaccination.

Coward, H., and P. Ratanakul. 1999. A Cross-Cultural Dialogue on Health Care Ethics. Waterloo, ON: Wilfrid Laurier University Press.

Davison, Janet. 2013. "Are We Living in Post-Religious Times?" CBC News. 20 March. http://www.cbc.ca/ news/canada/are-we-living-in-post-religious-times-1.1362828.

Dawson, L. 1999. "When Prophecy Fails and Faith Persists: A Theoretical Overview." Nova Religio 3 (1): 60-82. http://dx.doi.org/10.1525/nr.1999.3.1.60.

Deeks, S.L., G.H. Lim, R. Walton, J. Fediurek, F. Lam, C. Walker, J. Walters, and N.S. Crowcroft. 2014. "Prolonged Pertussis Outbreak in Ontario Originating in an Under-Immunized Religious Community." Canada Communicable Disease Report 40 (3): 42-9.

Dein, S. 2001. "What Really Happens when Prophecy Fails?" Sociology of Religion 62 (3): 383-401. http:// dx.doi.org/10.2307/3712356.

De Serres, G., F. Markowski, E. Toth, M. Landry, D. Auger, M. Mercier, P. Belanger, B. Turmel, H. Arruda, N. Boulianne et al. 2013. "Largest Measles Epidemic in North America in a Decade -Quebec, Canada, 2011: Contribution of Susceptibility, Serendipity, and Superspreading Events." Journal of Infectious Diseases 207 (6): 990-8. http://dx.doi.org/10.1093/infdis/jis923.

Dominus, Susan. 2011. "The Crash and Burn of and Autism Guru." New York Times. 11 April. http://www. nytimes.com/2011/04/24/magazine/mag-24Autism-t.html.

Dubé, E., C. Laberge, M. Guay, P. Bramadat, R. Roy, and J. Bettinger. 2013. "Vaccine Hesitancy: An Overview." Human Vaccines \& Immunotherapeutics 9 (8): 1763-73. http://dx.doi.org/10.4161/hv.24657.

Editors of The Lancet. 2010. "Retraction - Ileal-Lymphoid-Nodular Hyperplasia, Non- Specific Colitis, and Pervasive Developmental Disorder in Children." The Lancet 375 (9713): 445. 
Ernst, E. 2002. "Rise in Popularity of Complementary and Alternative Medicine: Reasons and Consequences for Vaccination." Vaccine 20: S90-3.

- 2005. "Is Homeopathy a Clinically Valuable Approach?" Trends in Pharmacological Sciences 26 (11): 547-8. http://dx.doi.org/10.1016/j.tips.2005.09.003.

Feldman-Savelsberg, P., F.T. Ndonko, and B. Schmidt-Ehry. 2000. "Sterilizing Vaccines or the Politics of the Womb: Retrospective Study of a Rumor in Cameroon." Medical Anthropology Quarterly 14 (2): 159-79. http://dx.doi.org/10.1525/maq.2000.14.2.159.

Festinger, L., H. Riecken, and S. Schachter. 1956. When Prophecy Fails: A Social and Psychological Study of a Modern Group that Predicted the Destruction of the World. Minneapolis: University of Minnesota Press.

Fisher, B.L. 2010. "Doctors, Judges, and Juries Hanging Their Own." Vaccine Awakening (Blog). 29 January. http://vaccineawakening.blogspot.ca/2010/01/vaccines-doctorjudges-juries-hanging.html.

Fisher, P. 2006. "Homeopathy and The Lancet." Evidence-Based Complementary and Alternative Medicine 3 (1): 145-7. http://dx.doi.org/10.1093/ecam/nek007.

Gardner, D. 2008. Risk: Why We Fear the Things We Shouldn't - and Put Ourselves in Greater Danger. Toronto: Emblem, McLelland and Stewart.

Gerber, J., and P. Offit. 2009. "Vaccines and Autism: A Tale of Shifting Hypotheses." Clinical Infectious Diseases $48(4): 456-61$.

Glatz, C. 2005. "Vatican: Refusing Vaccines Must be Weighed against Health Threats." Vatican-Vaccines XXXI, 2 August. http://www.catholicnews.com/data/stories/cns/0504240.htm.

Grabenstein, John D. 1999. "Moral Considerations with Certain Viral Vaccines." Christianity \& Pharmacy 2 (2): 3-6.

- 2013. "What the World's Religions Teach, Applied to Vaccines and Immune Globulins." Vaccine 31 (16): 2011-23. http://dx.doi.org/10.1016/j.vaccine.2013.02.026.

Graham-Harrison, E. 2012. "Afghan Gunmen Kill Polio Vaccination Worker in Latest Attack on Women." The Guardian, 5 December. https://www.theguardian.com/world/2012/dec/05/afghanistan-woman-health-volunteer-assassinated.

- 2013. "Immunisation Programme Halted in Nuristan Province." The Guardian, 12 March. https://www.theguardian.com/world/2013/mar/12/taliban-stoppingpolio-vaccinations-afghanistan.

Grande, David, Judy Shea, and Katrina Armstrong. 2011. "Pharmaceutical Industry Gifts to Physicians: Patient Beliefs and Trust in Physicians and the Health Care System." Journal of General Internal Medicine 27: 1760-3.

Guay, M., F. Gallagher, G. Petit, S. Ménard, P. Clément, and G. Boyer. 2009. Pourquoi les couvertures vaccinales chez les nourrissons de l'Estrie sont-elles sous-optimales? Sherbrooke: Centre de santé et de services sociaux - Institut universitaire de gériatrie de Sherbrooke.

Haidt, Jonathan. 2001. "The Emotional Dog and its Rational Tail: A Social Intuitionist Approach to Moral Judgment." Psychological Review 108 (4): 814-34. http://dx.doi.org/10.1037/0033-295X.108.4.814.

- 2012. The Righteous Mind: Why Good People Are Divided by Religion and Politics. New York: Allen Lane.

Hall, D., ed. 1997. Lived Religion in America: Toward a History of Practice. Princeton, NJ: Princeton University Press.

Healy, M.C., and L. Pickering. 2011. "How to Communicate with Vaccine-Hesitant Parents." Pediatrics 127 (Suppl): S127-33. http://dx.doi.org/10.1542/peds.2010-1722S. 
Hobson-West, P. 2003. "Understanding Vaccination Resistance: Moving Beyond Risk." Health Risk \& Society 5 (3): 273-83. http://dx.doi.org/10.1080/13698570310001606978.

Jegede, A.S. 2007. "What Led to the Nigerian Boycott of the Polio Vaccination Campaign?" Public Library of Science Medicine 4 (3): e73. http://dx.doi.org/10.1371/journal.pmed.0040073.

Kata, Anna. 2010. "A Postmodern Pandora's Box: Anti-Vaccination Misinformation on the Internet." Vaccine 28 (7): 1709-16. http://dx.doi.org/10.1016/j.vaccine.2009.12.022.

- 2012. "Anti-Vaccine Activists, Web 2.0, and the Postmodern Paradigm - An Overview of Tactics and Tropes Used Online by the Anti-Vaccination Movement." Vaccine 30 (25): 3778-89. http://dx.doi.org/10.1016/j.vaccine.2011.11.112.

Kennedy, A.M., and D.A. Gust. 2008. "Measles Outbreak Associated with a Church Congregation: A Study of Immunization Attitudes of Congregation Members." Public Health Reports 123 (2): 126-34.

Kudha-Bukhsh, A.R. 2003. "Towards Understanding the Molecular Mechanisms of Action of Homeopathic Drugs: An Overview." Molecular and Cellular Biochemistry 253 (1/2): 339-45. http://dx.doi.org/10.1023/A:1026048907739.

Latour, B. 2009. On the Modern Cult of the Factish Gods. Translated by H. MacLean and C. Porter. Durham, NC: Duke University Press.

Leask, J., P. Kinnersley, C. Jackson, F. Cheater, H. Bedford, and G. Rowles. 2012. "Communicating with Parents about Vaccination: A Framework for Health Professionals." BMC Pediatrics 12 (1): 154. http:// dx.doi.org/10.1186/1471-2431-12-154.

Lefebvre, Solange. 2008. "The Francophone Roman Catholic Church." In Christianity and Ethnicity in Canada, edited by P. Bramadat and D. Seljak, 101-37. Toronto: University of Toronto Press. http://dx.doi. org/10.3138/9781442687622-005.

Macdonald, Neil. 2016. "The 'Post-Truth' President Flattens Fact-Obsessed Media." CBC News. 28 November. http://www.cbc.ca/news/opinion/post-truthpresident-1.3871021.

MacDonald, P.F. 2007. "The MMR Vaccine Controversy - Winners, Losers, Impact and Challenges." British Journal of Infection Control 8 (1): 18-22. http://dx.doi.org/10.1177/14690446070080010901.

Maki, Allan. 2014. "Measles in Canada: Why this infectious disease is spreading." Globe and Mail, 8 April. http://www.theglobeandmail.com/life/health-andfitness/health/measles-in-canada-why-this-infectious-diseases-is-spreading/article17866080/?page=all.

Mnookin, S. 2012. The Panic Virus: The True Story behind the Vaccine-Autism Controversy. New York: Simon and Schuster.

Moore, R., and S. McClean, eds. 2010. Folk Healing and Health Care Practices in Britain and Ireland: Stethoscopes, Wands and Crystals. Oxford: Berghahn Press.

Moutsiakis, D.L., and N.P. Chin. 2007. "Why Blacks Do Not Take Part in HIV Vaccine Trials." Journal of the National Medical Association 99 (3): 254-7.

Offit, P. 2011. Deadly Choices: How the Anti-Vaccine Movement Threatens Us All. New York: Basic Books.

Omer, S.B., D. Salmon, W.A. Orenstein, P. deHart, and N. Halsey. 2009. "Vaccine Refusal, Mandatory Immunization, and the Risks of Vaccine-Preventable Diseases." New England Journal of Medicine 360: 1981-8.

Oostvogel, P.M., J.K. Van Wijngaarden, H. Van der Avoort, M.N. Mulders, M. Conyn-van Spaendonck, H.C. Rümke, G. van Steenis, and A.M. van Loon. 1994. "Poliomyelitis Outbreak in an Unvaccinated Community in the Netherlands, 1992-93." The Lancet 344 (8923): 665-70. http://dx.doi.org/10.1016/S01406736(94)92097-5. 
Orsi, R. 2004. Between Heaven and Earth: The Religious Worlds People Make and the Scholars Who Study Them. Princeton, NJ: Princeton University Press.

Osterholm, M.T., N.S. Kelley, A. Sommer, and E.A. Belongia. 2012. "Efficacy and Effectiveness of Influenza Vaccines: A Systematic Review and Meta-Analysis." Lancet Infectious Diseases 12 (1): 36-44. http:// dx.doi.org/10.1016/S1473-3099(11)70295-X.

Paper, J., L.C. Paper, and D. Lai. 2009. "The Chinese in Canada: Their Unrecognized Religion." In Religion and Ethnicity in Canada, edited by Paul Bramadat and David Seljak, 89-110. Toronto: University of Toronto Press.

Poland, G.A., R.M. Jacobson, and I.G. Ovsyannikova. 2009. "Trends Affecting the Future of Vaccine Development and Delivery: The Role of Demographics, Regulatory Science, the Anti-Vaccine Movement, and Vaccinomics." Vaccine 27 (25-6): 3240-4. http://dx.doi.org/10.1016/j.vaccine.2009.01.069.

Poltorak, M., M. Leach, J. Fairhead, and J. Cassell. 2005. "'MMR Talk' and Vaccination Choices: An Ethnographic Study in Brighton." Social Science \& Medicine 61 (3): 709-19. http://dx.doi.org/10.1016/j.socscimed.2004.12.014.

Pontifical Academy of Life. 2005. "Moral Reflections on Vaccines Prepared From Cells Derived From Aborted Human Foetuses." National Catholic Bioethics Quarterly 6 (3): 541-47. http://www.ncbi.nlm.nih. gov/pubmed/17091557.

Riley, D., M. Fischer, B. Singh, M. Haidvogl, and M. Heger. 2001. "Homeopathy and Conventional Medicine: An Outcomes Study Comparing Effectiveness in a Primary Care Setting." Journal of Alternative and Complementary Medicine 7 (2): 149-59. http://dx.doi.org/10.1089/107555301750164226.

Ruijs, W., J. Hautvast, K. van der Velden, S. de Vos, H. Knippenberg, and M. Hulscher. 2011. "Religious Subgroups Influencing Vaccination Coverage in the Dutch Bible Belt: An Ecological Study." BMC Public Health 11 (1): 102. http://dx.doi.org/10.1186/1471-2458-11-102.

Salmon, D.A., L.H. Moulton, S.B. Omer, M.P. DeHart, S. Stokley, and N.A. Halsey. 1999. "Health Consequences of Religious and Philosophical Exemptions from Immunization Laws Individual and Societal Risk of Measles." Journal of the American Medical Association 281 (1): 47-53.

Scullard, P., C. Peacock, and P. Davies. 2010. "Googling Children's Health: Reliability of Medical Advice on the Internet." Archives of Disease in Childhood 95 (8): 580-2. http://dx.doi.org/10.1136/adc.2009.168856.

Shang, A., K. Huwiler-Müntener, L. Nartey, P. Jüni, S. Dörig, J.A.C. Sterne, D. Pewsner, and M. Egger. 2005. "Are the Clinical Effects of Homoeopathy Placebo Effects? A Comparative Study of Placebo-Controlled Trials of Homoeopathy and Allopathy." The Lancet 366 (9487): 726-32. http://dx.doi.org/10.1016/ S0140-6736(05)67177-2.

Shellnutt, Kate. 2016. "Trump Elected President, Thanks to 4 in 5 White Evangelicals." Christianity Today. 9 November 2016. http://www.christianitytoday.com/gleanings/2016/november/trump-elected-president-thanks-to-4-in-5-white-evangelicals.html.

Shnier, A., J. Lexchin, B. Mintzes, A. Jutel, and K. Holloway. 2013. "Too Few, Too Weak: Conflict of Interest Policies at Canadian Medical Schools." PLoS One 8 (7):e68633. http://dx.doi.org/10.1371/journal. pone.0068633.

Snow, D., and R. Machalek. 1982. "On the Presumed Fragility of Unconventional Beliefs." Journal for the Scientific Study of Religion 21 (1): 15-26. http://dx.doi.org/10.2307/1385566.

Spier, R.E. 2001. "Perception of Risk of Vaccine Adverse Events: A Historical Perspective." Vaccine 20: S7884. http://dx.doi.org/10.1016/S0264-410X(01)00306-1.

Stackhouse, John Jr. 1993. Canadian Evangelicalism in the Twentieth Century: An Introduction to Its Character. Toronto: University of Toronto Press.

Stark, R. 1998. "The Rise and Fall of Christian Science." Journal of Contemporary Religion 13 (2): 189-214. http://dx.doi.org/10.1080/13537909808580830. 
Statistics Canada. 2003. "Religions in Canada." 2001 Census: Analysis Series. Statistics Canada Catalogue no. 96F0030XIE2001015. Ottawa. 13 May.

- 2011. National Household Survey Data Tables. Statistics Canada Catalogue no. 99-010-X2011032. Ottawa: Released 8 May 2013.

- 2014. General Social Survey [Canada]: Cycles 1-26 (1985-2012). Public use microdata files.

Streefland, P.H. 2001. "Public Doubts about Vaccination Safety and Resistance Against Vaccination." Health Policy 55 (3): 159-72. http://dx.doi.org/10.1016/S0168-8510(00)00132-9.

Streefland, P., A. Chowdhury, and P. Ramos-Jimenez. 1999. "Patterns of Vaccination Acceptance." Social Science \& Medicine 49 (12): 1705-16. http://dx.doi.org/10.1016/S0277-9536(99)00239-7.

Taylor, C. 1992. The Ethics of Authenticity. Boston: Harvard University Press.

Thagard, P. 2010. "Does Everything Happen for a Reason?" Psychology Today. 11 February. https://www. psychologytoday.com/blog/hot-thought/201002/does-everything-happen-reason-0.

Tickner, S., P.J. Leman, and A. Woodcock. 2006. "Factors Underlying Suboptimal Childhood Immunization." Vaccine 24 (49-50): 7030-6. http://dx.doi.org/10.1016/j.vaccine.2006.06.060.

Tumminia, D. 1998. "How Prophecy Never Fails: Interpretive Reason in a Flying Saucer Group." Journal of Contemporary Religion 59 (2): 157-70.

UNICEF. 1997. Combating Antivaccination Rumors: Lessons Learned from Case Studies in East Africa. Nairobi, Kenya: UNICEF. http://www.path.org/vaccineresources/files/Combatting_Antivac_Rumors_ UNICEF.pdf.

UNICEF/WHO. 2012. Immunization Summary: A Statistical Reference Containing Data through 2010. New York: UNICEF/World Health Organization. http://www.nitag-resource.org/uploads/media/defauIt/0001/02/933358b68002a47d8cc5d99b8327be4f3a345fa7.pdf.

Vaccine Risk Awareness Network (VRAN). 1984. "A Brief to the Ministry of Health, Government of Ontario: The Committee Against Compulsory Vaccination." Vaccine Risk Awareness Network. Accessed 7 January 2017, http://vran.com.s9135.gridserver.com/legacy/philosophy/history.htm.

-2005. "Rubella Outbreak in Ontario - VRAN Information Bulletin." Vaccination Risk Awareness Network. May. Accessed 7 January 2017, http://vran.com.s9135.gridserver.com/legacy/vaccines/mmr/rub-bull. htm.

Vitello, Paul. 2010. "Christian Science Church Seeks Truce with Modern Medicine." New York Times. 23 March. http://www.nytimes.com/2010/03/24/nyregion/24heal.html?ref=topics.

Warraich, H.J. 2009. "Religious Opposition to Polio Vaccination." Emerging Infectious Diseases 15 (6): 978. http://dx.doi.org/10.3201/eid1506.090087.

Wolfe, R.M., L.K. Sharp, and M.S. Lipsky. 2002. "Content and Design Attributes of Anti-Vaccination Web Sites." Journal of the American Medical Association 287 (24): 3245-8. http://dx.doi.org/10.1001/ jama.287.24.3245.

Woodhead, L., and P. Heelas. 2005. The Spiritual Revolution: Why Religion Is Giving Way to Spirituality. Oxford: Blackwell.

Wuthnow, R. 2000. After Heaven: Spirituality in America since the 1950s. Los Angeles: University of California Press.

Zuzak, T.J., I. Zuzak-Siegrist, L. Rist, G. Staubli, and A.P. Simoes-Wust. 2008. "Attitudes towards Vaccination: Users of Complementary and Alternative Medicine Versus Non-Users." Swiss Medical Weekly 138 (47-8): 713-18. 